ARTICLE

https://doi.org/10.1038/s41467-019-10676-1

\title{
IL-33-mediated mast cell activation promotes gastric cancer through macrophage mobilization
}

\author{
Moritz F. Eissmann ${ }^{1}$, Christine Dijkstra ${ }^{1}$, Andrew Jarnicki ${ }^{10}$, Toby Phesse (1) 1,6, Jamina Brunnberg ${ }^{1,7}$, \\ Ashleigh R. Poh', Nima Etemadi (1) 1,8, Evelyn Tsantikos², Stefan Thiem', Nicholas D. Huntington', \\ Margaret L. Hibbs ${ }^{2}$, Alex Boussioutas ${ }^{4}$, Michele A. Grimbaldeston ${ }^{5,9}$, Michael Buchert ${ }^{1}$, \\ Robert J.J. O'Donoghue ${ }^{1,10}$, Frederick Masson ${ }^{1,11}$ \& Matthias Ernst ${ }^{1}{ }^{1}$
}

The contribution of mast cells in the microenvironment of solid malignancies remains controversial. Here we functionally assess the impact of tumor-adjacent, submucosal mast cell accumulation in murine and human intestinal-type gastric cancer. We find that genetic ablation or therapeutic inactivation of mast cells suppresses accumulation of tumorassociated macrophages, reduces tumor cell proliferation and angiogenesis, and diminishes tumor burden. Mast cells are activated by interleukin (IL)-33, an alarmin produced by the tumor epithelium in response to the inflammatory cytokine IL-11, which is required for the growth of gastric cancers in mice. Accordingly, ablation of the cognate IL-33 receptor St2 limits tumor growth, and reduces mast cell-dependent production and release of the macrophage-attracting factors $\mathrm{Csf2}, \mathrm{Ccl} 3$, and 116 . Conversely, genetic or therapeutic macrophage depletion reduces tumor burden without affecting mast cell abundance. Therefore, tumor-derived IL-33 sustains a mast cell and macrophage-dependent signaling cascade that is amenable for the treatment of gastric cancer.

\footnotetext{
${ }^{1}$ Cancer and Inflammation Laboratory, Olivia Newton-John Cancer Research Institute and School of Cancer Medicine, La Trobe University, Heidelberg, VIC 3084, Australia. ${ }^{2}$ Department of Immunology and Pathology, Monash University, Melbourne, VIC 3004, Australia. ${ }^{3}$ Molecular Immunology Division, The Walter and Eliza Hall Institute of Medical Research, and Department of Medical Biology, University of Melbourne, Melbourne, VIC 3052, Australia.

${ }^{4}$ Department of Medicine, University of Melbourne, Melbourne, VIC 3050, Australia. ${ }^{5}$ Centre for Cancer Biology, University of South Australia and SA Pathology, Adelaide, SA 5000, Australia. ${ }^{6}$ Present address: Cell Signaling and Cancer Laboratory, European Cancer Stem Cell Research Institute and Cardiff University, Cardiff CF24 4HQ, UK. ${ }^{7}$ Present address: Institute of Biochemistry, Goethe University Frankfurt, Frankfurt am Main, 60438 Frankfurt, Germany. ${ }^{8}$ Present address: Cell Signalling and Cell Death Division, Walter and Eliza Hall Institute of Medical Research, and Department of Medical Biology, University of Melbourne, Melbourne, VIC 3052, Australia. ${ }^{9}$ Present address: OMNI-Biomarker Development, Genentech Inc., South San Francisco, CA 94080, USA ${ }^{10}$ Present address: Department of Pharmacology and Therapeutics, University of Melbourne, Melbourne, VIC 3010, Australia. ${ }^{11}$ Present address: Team 5, Centre of Physiopathology Toulouse-Purpan, INSERM UMR 1043/CNRS UMR 5282, University Toulouse III, CHU Purpan, 31024 Toulouse, France. Correspondence and requests for materials should be addressed to M.E. (email: Matthias.ernst@onjcri.org.au)
} 
T he interactions between cancer cells and their microenvironment can result in tumor progression as well as suppression and/or eradication of cancers ${ }^{1}$. Besides extracellular matrix and stromal cells, the tumor microenvironment is composed of immune cells of the adaptive and innate immune system, with the latter comprising neutrophils, macrophages, mast cells, myeloid-derived suppressor cells, dendritic cells, innate lymphocytes, and natural killer cells. Often T-lymphocytes and tumor-associated macrophages (TAMs) account for the most abundant immune cell populations infiltrating established tumors ${ }^{2,3}$

Mast cells are long-lived secretory cells of hematopoietic origin that function as sentinels by responding to changes in their environment ${ }^{4}$. They respond to extrinsic signals through a multitude of cell surface receptors to secrete histamine and proteases from prestored sources in cytoplasmic granules ${ }^{5}$, alongside newly synthesized inflammatory mediators ${ }^{4}$. Accordingly, mast cells can be part of innate and adaptive immune responses and therefore contribute to various pathophysiological conditions ${ }^{6}$. In cancer, the presence of mast cells, both at the tumor periphery and in the tumor core can correlate with disease progression, increased metastasis and reduced survival of patients with melanoma ${ }^{7}$, prostate cancer $^{8}$, pancreatic adenocarcinoma ${ }^{9}$, squamous cell carcinoma ${ }^{10}$, and gastric cancer ${ }^{11,12}$. However in other solid malignancies, mast cells have been associated with more favorable outcomes ${ }^{13-15}$. The conflicting nature of these correlative findings are reminiscent of contradictory functional observations in mouse models claiming that mast cells are required for pancreatic islets tumorigenesis ${ }^{16}$, while pancreatic adenocarcinoma occurs in a tumor microenvironment devoid of mast cells ${ }^{17}$.

Interleukin (IL)-33 is a danger-associated signal that can serve as a molecular alarmin when released upon necroptotic and necrotic cell death including death of cancer cells ${ }^{18-21}$. However, cell death-independent IL-33 release can also occur ${ }^{22,23}$. IL-33 signals through the heterodimeric ST2 receptor, encoded by Illrl1 gene, which is constitutively expressed on the surface of some innate immune cells including mast cells ${ }^{24}$, innate lymphoid cells type 2 (ILC2) $)^{25}$, and regulatory T-cells (Treg) ${ }^{26,27}$. IL-33/ ST2 signaling is involved in triggering innate immune responses upon parasite and viral infections, and has been identified as an important mast cell activating factor ${ }^{24,28}$ in the context of allergy ${ }^{29}$. Furthermore, elevated IL-33 expression was associated with poor outcomes in patients with gliomas ${ }^{30}$, ovarian ${ }^{31}$, as well as head and neck cancers ${ }^{32}$. However, predicting the outcome of IL-33/ST2 signaling in malignancies remains uncertain with both tumor promoting as well as tumor restricting activities being reported in knockout mouse models ${ }^{33-36}$

Here, we employ preclinically validated mouse models of gastric cancer and corresponding patient biopsies to functionally elucidate the role of mast cells during gastric tumorigenesis. Our genetic analysis reveals a linear signaling axis initiated by tumor epithelial-derived IL-33 that activates mast cells to produce a chemotactic cytokine expression signature. These factors promote the accumulation of TAMs, which in turn sustain tumor angiogenesis and growth in mice. In gastric cancer patients, this mast cell activation signature, alongside markers for tumor-associated macrophages, correlates with decreased patient survival. Our findings delineate an IL-33/mast cell/macrophage axis, which affords a clinical opportunity for the treatment of gastric cancer.

\section{Results}

Increased mast cell density in human intestinal-type gastric cancer and in corresponding mouse models. In order to characterize the role of mast cells in gastric cancer, we initially investigated the mast cell frequency in $g p 130^{\mathrm{FF}}$ mice, a preclinically validated model for spontaneously occurring intestinal-type gastric cancer ${ }^{37,38}$. Gastric tumors in $g p 130^{\mathrm{FF}}$ mice, which harbor a knock in germline mutation in the shared IL-6/IL-11 receptor subunit gp130, arise from excessive IL-6/IL11 dependent STAT3 activity; these tumors remain associated with chronic inflammation and immune cell infiltration. We used toluidine blue stains to quantify mast cells, and observed profound accumulation of mast cells in the submucosa of the gastric antrum in $g p 130^{\mathrm{FF}}$ mice compared to wild-type mice. Importantly, mast cells in the submucosa adjacent to gastric tumors were more abundant than the mast cells in the normal antrumassociated submucosa of $g p 130^{\mathrm{FF}}$ mice (Fig. 1a, b). In addition, mast cell numbers in $g p 130^{\mathrm{FF}}$; Stat $3^{+/}$compound mutant mice, which neither develop gastric tumors nor the other paninflammatory changes observed in their Stat3-proficient $g p 130^{\mathrm{FF}} ;$ Stat $^{+/+}$littermates $^{39,40}$, remained comparable to those in wild-type mice.

To ascertain that submucosal mast cell accumulation correlated with tumorigenesis independently of the nature of the oncogenic driver mutation, we also assessed mast cell distribution in $\mathrm{Tg}$ (Tff1-CreERT2); Pik3ca ${ }^{\mathrm{H} 1047 \mathrm{R} /+}$; Pten ${ }^{\mathrm{f} / \mathrm{fl}}$ mice. In this model, conditional activation of a pan-glandular gastric epitheliumspecific Cre-recombinase induces aberrant activation of phosphatidylinositol 3-kinase (PI3K), a pathway frequently mutated in human gastric cancer. Akin to our observations in $g p 130^{\mathrm{FF}}$ mice, mast cells also accumulated in the submucosa of tumors in $\mathrm{Tg}$ (Tff1-CreERT2); Pik3ca ${ }^{\mathrm{H} 1047 \mathrm{R} /+} ;$ Pten $^{\mathrm{f} / \mathrm{fl}}$ mice (Supplementary Fig. 1a, b). Strikingly, toluidine blue, alcian blue/safranin O, and May-Grunwald-Giemsa stains for granular content all consistently revealed that mast cells localized to the submucosal layers of the antrum and body of the stomach, rather than within the tumors (Supplementary Fig. 1c).

We next assessed the stromal abundance of mast cells in human biopsies collected from patients with either histopathologically classified diffuse or intestinal-type gastric cancer, or patients with preneoplastic intestinal metaplasia or chronic gastritis. Similar to our observation in mice, we observed a profound submucosal accumulation of toluidine-blue positive mast cells in intestinal-type gastric cancer. Strikingly, mast cells numbers were not elevated in the submucosa of patients with gastritis or intestinal metaplasia (Fig. 1c, d). Collectively, our results show increased mast cell abundance in the tumor-adjacent submucosa of intestinal-type gastric adenocarcinoma in human and mice, irrespective of the nature of the oncogenic driver mutation(s).

Mast cell deficiency diminishes $g p 130^{\mathrm{FF}}$-driven tumor growth Having established a reproducible correlation between submucosal mast cell density and gastric cancer in humans and mice, we next determined the functional role of mast cells during tumor initiation and progression. For this we exploited the $g p 130^{\mathrm{FF}}$ model because of its high disease concordance with the human GC subtype, reproducible penetrance, onset, and progression of gastric tumorigenesis.

In order to establish whether submucosal mast cell accumulation preceded tumorigenesis, we analyzed stomach sections of 4week-old $g p 130^{\mathrm{FF}}$ mice prior to onset of tumor formation. We detected increased mast cell density in the gastric submucosa of these tumor-free $g p 130^{\mathrm{FF}}$ mice when compared to the submucosa of age-matched WT littermates (Supplementary Fig. 2a). To functionally determine whether mast cells indeed promote gastric tumor growth, we crossed $g p 130^{F F}$ mice with mast cell-deficient $\mathrm{C} 57 \mathrm{BL} / 6 \mathrm{c}-\mathrm{Kit} \mathrm{W}^{\mathrm{W}-\mathrm{sh} / \mathrm{W} \text {-sh }}$ mice carrying an inversion of the $5^{\prime}$ regulatory region of the $c$-Kit gene that results in hypomorphic expression of the corresponding stem cell factor receptor protein. 

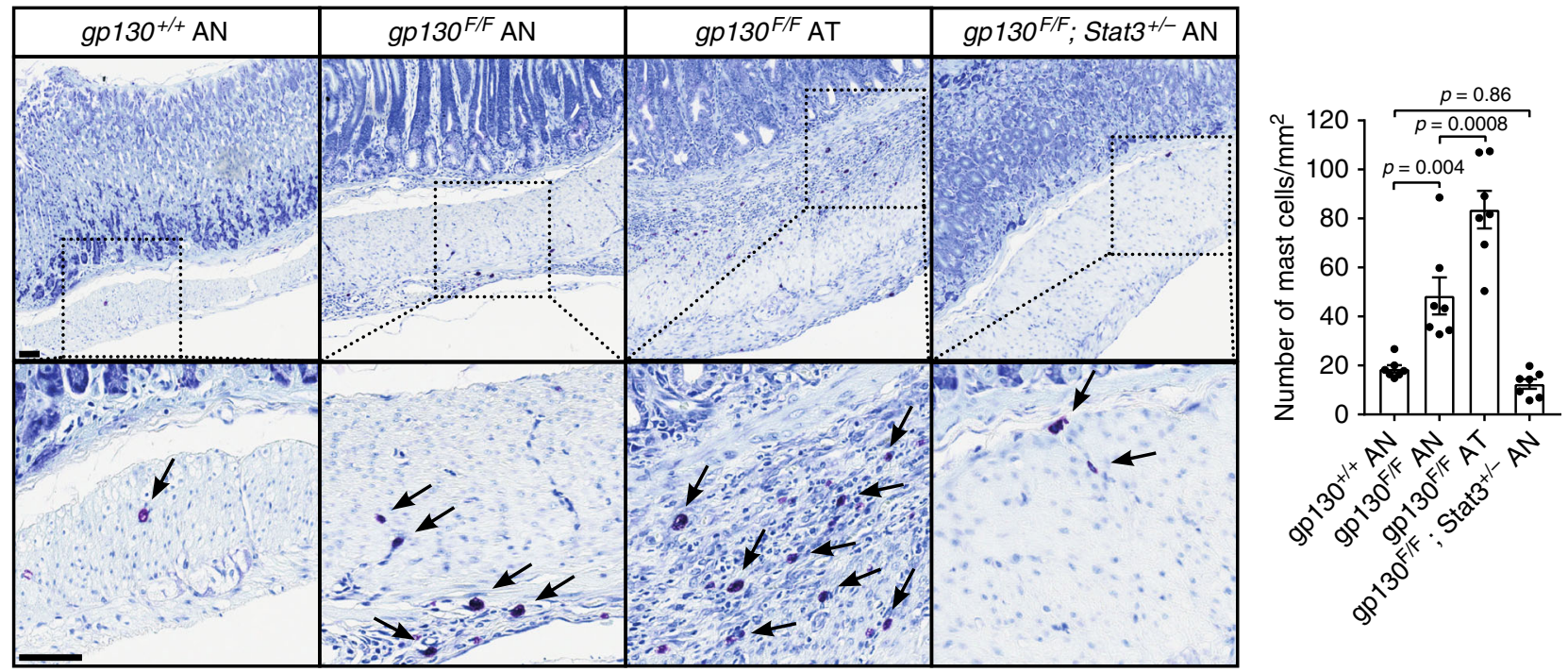

C

Mast cells in human gastric cancer
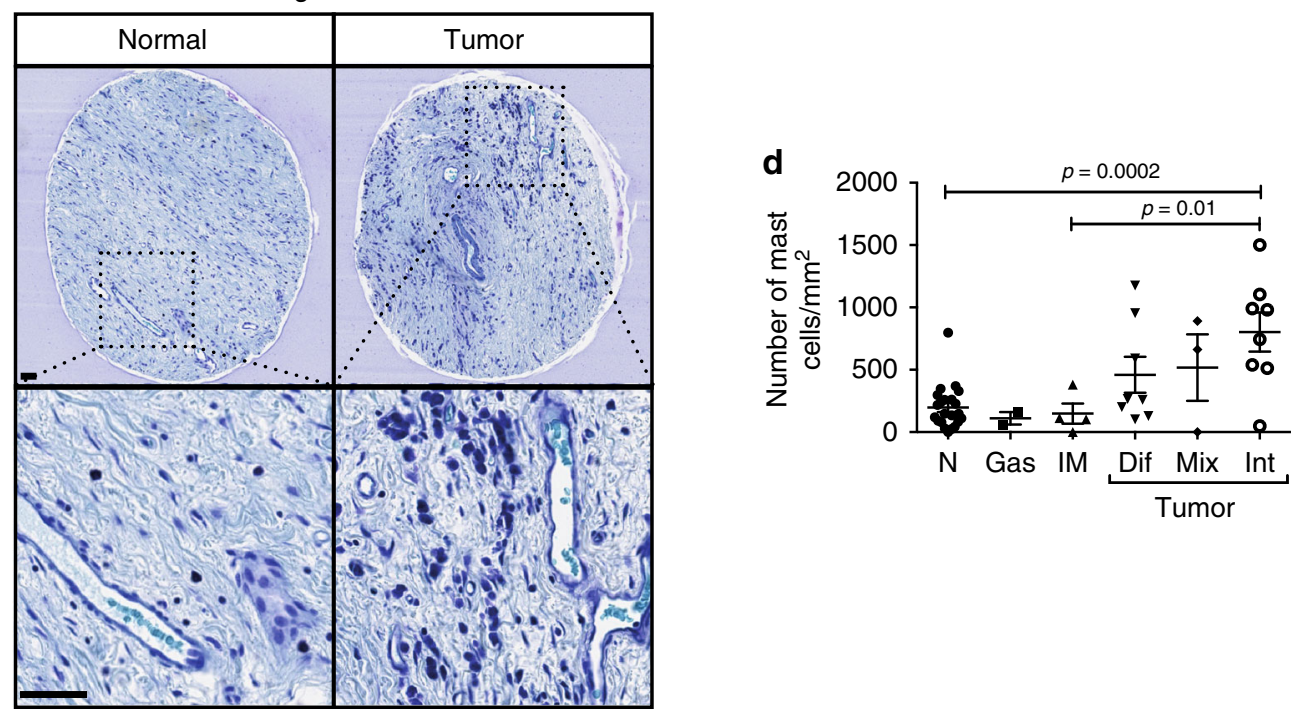

Fig. 1 Submucosal mast cell numbers are increased in gastric cancer in mice and humans. a Representative cross sections of stomachs of 100 day old gp130 mutant mice of the indicated genotype and stained with toluidine blue showing the affected antrum (AN) and antral tumor (AT), respectively. Mast cells appear purple (arrows). Scale bars $=50 \mu \mathrm{m}$. b Quantification of submucosal mast cell in sections depicted in (a). $n=7$ mice per cohort obtained from two independent experiments, one-way ANOVA F (DFn = degree of freedom nominator, Dfd = degree of freedom denominator) $=34.96(3,24)$.

c Representative sections of toluidine blue-stained biopsy cores of human gastric cancer (GC) and adjacent submucosa and of normal stomach submucosa. Scale bars $=50 \mu \mathrm{m}$. d Quantification of submucosal mast cell in sections depicted in c. Each symbol represents an individual patient biopsy from submucosa of normal ( $N ; n=22)$, gastritis (Gas; $n=2)$, intestinal metaplasia (IM; $n=4$ ), diffuse (Dif; $n=8)$, mixed (Mix; $n=3$ ), and intestinal-type $($ Int; $n=8)$ gastric cancer. One-way ANOVA F $(D F n, D f d)=5.809(5,41)$. Data are represented as mean \pm SEM, with $p<0.05$ considered significant. Source data are provided as a Source Data file. See also related Supplementary Fig. 1

Accordingly, $c$-Kit $\mathrm{W}$-sh $/ \mathrm{W}$-sh mice exhibit a substantial reduction of mast cells in all tissues ${ }^{41-43}$, but do not suffer from anemia, sterility and lethality associated with complete c-Kit deficiency ${ }^{41}$. Likewise, we did not observe differences in the peripheral blood composition between $g p 130^{\mathrm{FF}}$ and $g p 130^{\mathrm{FF}} ; c$-Kit ${ }^{\mathrm{W}-\mathrm{sh} / \mathrm{W} \text {-sh }}$ compound mutants and we confirmed that EpCAM positive tumor cells lack c-Kit expression (Supplementary Figs. 2b, c). However, $g p 130^{\mathrm{FF}} ; c-K i t^{\mathrm{W}-\mathrm{sh} / \mathrm{W}-\mathrm{sh}}$ compound mice had significantly smaller and fewer tumors than their age and sex-matched $g p 130^{\mathrm{FF}}$ littermates (Fig. 2a-c). This reduction in tumor burden coincided with reduced tumor cell proliferation rather than increased apoptosis, and was associated with a reduction of $\mathrm{CD}^{+} 1^{+}$endothelial cells (Fig. 2d and Supplementary Fig. 2d).

To exclude that the anticancer effect of the $c$-Kit $\mathrm{W}$-sh hypomorphic allele was not in part mediated by hematopoietic cells other than mast cells, we generated mast cell-deficient gp130FF; Cpa3-Cre; Mcl1 ${ }^{f l f l}$ gastric cancer mice, where mast cellspecific carboxypeptidase A3 (Cpa3) promoter driven Cre recombinase activity leads to the deletion of $\mathrm{Mcll}$ prosurvival gene. As a consequence Cpa3-Cre; $\mathrm{Mcll}^{f l / f l}$ mice retain less than $10 \%$ mast cells and have reduced numbers of basophils, while all other hematopoietic cell populations remain unaffected ${ }^{44}$. We 

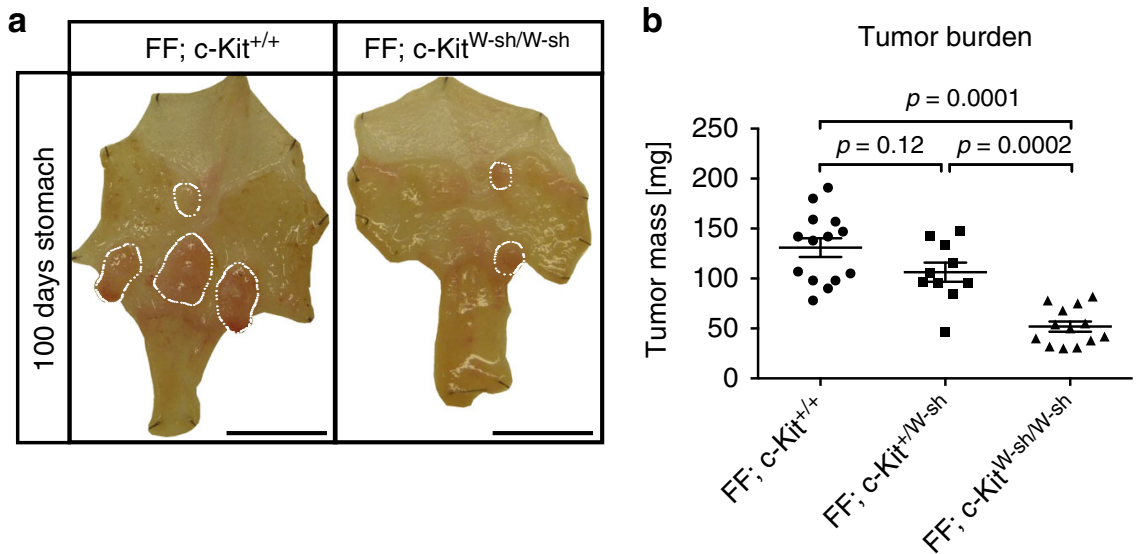

C Tumor numbers

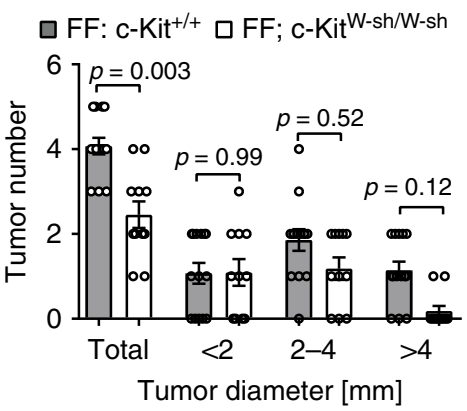

d $\quad \mathrm{FF} ; \mathrm{c}-\mathrm{Kit}^{+/+} \mathrm{aFF} ; \mathrm{c}-\mathrm{Kit} \mathrm{W}^{\mathrm{W}-\mathrm{sh} / \mathrm{W}-\mathrm{sh}}$
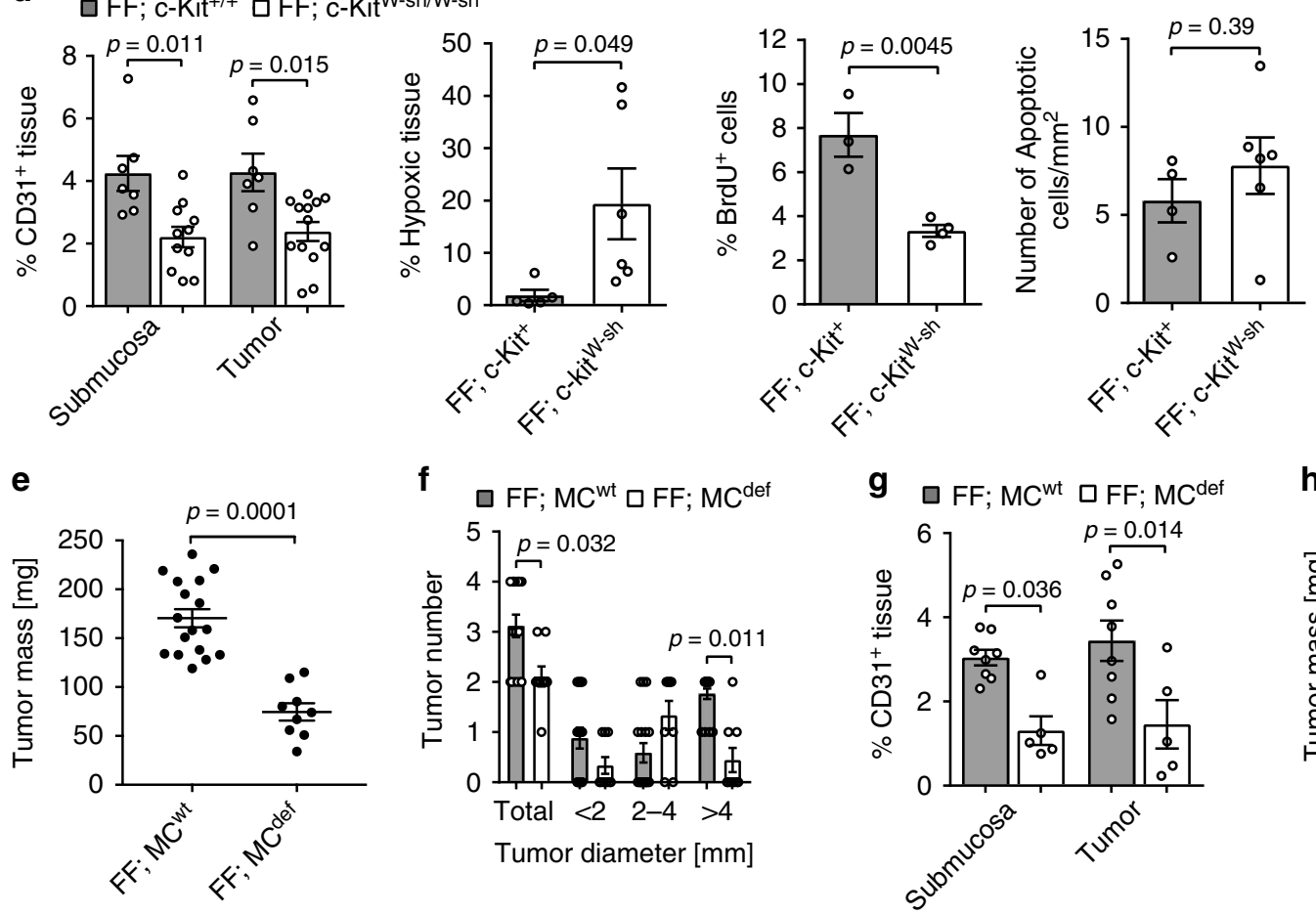

h

Fig. 2 Gastric tumor burden is reduced in mast cell-deficient gp130FF tumor mice. a Representative whole mounts of pinned out stomachs, from 100-dayold $g p 130^{\mathrm{FF}} ; \mathrm{c}-\mathrm{Kit}^{+/+}$and mast cell-deficient $\mathrm{gp} 13 \mathrm{O}^{\mathrm{FF}} ; \mathrm{c}-\mathrm{Kit} \mathrm{W}$-sh/W-sh mice. Scale bars $=1 \mathrm{~mm}$. b Quantification of total tumor burden per mouse as in (a). Each symbol represents an individual mouse. One-way ANOVA F (DFn, Dfd) $=25.97(2,24)$. c Enumeration of total tumor number and tumor size distribution as in a from gp130FF; c-Kit $+/+(n=14)$ and gp130FF; c-Kit W-sh/W-sh mice $(n=11)$. One-way ANOVA F (DFn, Dfd) $=24.59(7,92)$.

d Quantification of CD31, Hypoxyprobe (hypoxia), BrdU (proliferation), or ApopTag (apoptosis) on immunostained gastric tumors sections. Number of mice (n) for CD31: FF; c-Kit ${ }^{+/+} n=7$, FF; c-Kit W-sh/W-sh $n=11$ with ANOVA F (DFn, Dfd) = 7.075 (3, 34); Hypoxyprobe: FF; c-Kit ${ }^{+} n=5$, FF; c-Kit ${ }^{2}$-sh $n=6$ with $t$-test + Welch correction's $t(d f)=2.55$ (5.256); BrdU: FF; c-Kit ${ }^{+} n=3$, FF; c-KitW-sh $n=4$ with $t$-test's t (df) $=4.905$ (5); Apoptosis: FF; c-Kit ${ }^{+}$ $n=4$, FF; c-Kit W-sh $n=6$ with $t$-test's $t(d f)=0.89$ (8). e Quantification of total tumor burden per mouse of mast FF; MCwt (genotypes: FF; Cpa3-Cre ${ }^{\text {neg; }}$

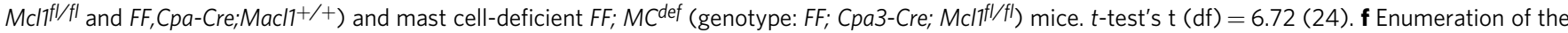
tumor number per mouse of FF; MCwt $(n=17$ mice) and mast cell-deficient FF; MCdef ( $n=9 \mathrm{FF}$; Cpa3-Cre; Mclffl/fl mice). One-way ANOVA F (DFn, Dfd) $=$ $23.25(7,96)$. $\mathbf{g}$ CD31 angiogenic staining quantification of stomachs from (e, f). FF; MCwt $(n=8$ mice) and FF; MCdef ( $n=5)$ with one-way ANOVA F (DFn, $\mathrm{Dfd})=6.79(3,22) . \mathbf{h}$ Quantification of total tumor burden in $g p 130^{\mathrm{FF}}$ mice after 6 weeks administration of cromolyn (mast cell degranulation inhibitor) or vehicle. Each symbol represents an individual mouse and data was generated in three independent experiments. $t$-test's $p$ value is shown and $t$ (df) $=2.313$ (18). Data are represented as mean \pm SEM, with $p$ values $p<0.05$, being considered significant. Source data are provided as a Source Data file. See also related Supplementary Fig 2

confirmed that $g p 130^{F F}$; Cpa3-Cre; Mcl1 fl/fl mutant mice lack mast cells in their stomachs, while their $\mathrm{gp} 130^{\mathrm{FF}}$; $\mathrm{Cpa3}-\mathrm{Cre}$; $\mathrm{Mcll}^{+/+}$ littermates display normal mast cell density (Supplementary Fig. 2e). Importantly, mast cell-deficient $g p 130^{F F}$;Cpa3Cre; Mcliflfl mice had significantly reduced tumor mass and tumor number compared to their mast cell-proficient controls (Fig. 2e, f), and this observation coincided with reduced angiogenic vessel density in the tumors of $g p 130^{F F}$; Cpa3-Cre; Mcl1 flffl mice (Fig. 2g).

To assess whether therapeutic mast cell manipulation could reduce the burden of established tumors, we exploited sodium cromoglycate (cromolyn) as a blocking agent for mast cell degranulation in patients. We treated tumor-bearing $g p 130^{\mathrm{FF}}$ mice for 6 weeks with cromolyn, which significantly decreased 
tumor burden when compared to vehicle-treated mice (Supplementary Fig. $2 \mathrm{f}$ and Fig. 2h). Akin to our observations genetic observations in $g p 130^{\mathrm{FF}} ; c-K i t^{\mathrm{W}-\mathrm{sh} / \mathrm{W} \text {-sh }}$ and $g p 130^{\mathrm{FF}}$; Cpa3Cre; $M c l f^{f l / f l}$ mice, cromolyn treatment of $g p 130^{\mathrm{FF}}$ mice also reduced macrophage accumulation, tumor angiogenesis and proliferation (Supplementary Fig. 2g). These findings demonstrate the importance of mast cells and their released products for gastric tumor growth and suggest that mast cells promote tumor proliferation through mechanisms involving angiogenesis.

In the $g p 130^{F F}$ mutant mice all cells harbor the $g p 130^{F}$ mutation. When expressed, the $g p 130^{F}$ mutant protein increases Stat 3 signaling in response to IL- 6 family cytokines. Because mast cells express the gp130 coreceptor and can respond to IL-6 family cytokines $^{45,46}$, we next excluded the possibility that the $g p 130^{\mathrm{F}}$ allele in mast cells may augment their tumor-promoting ability. For this, we reconstituted the hematopoietic compartment of 6week-old lethally irradiated $g p 130^{\mathrm{FF}}$ hosts with the bone marrow from WT $\left(g p 130^{+/+}\right)$or $g p 130^{F F}$ mice (Supplementary Fig. 2h). Indeed, WT $\rightarrow g p 130^{\mathrm{FF}}$ and $g p 130^{\mathrm{FF}} \rightarrow g p 130^{\mathrm{FF}}$ bone marrow chimeras showed not only similar mast cell frequencies in the gastric submucosa but also comparable tumor burden, and macrophage infiltration in the submucosa and tumor core (Supplementary Fig. 2i). These results indicate that the systemic presence of the $g p 130^{F F}$ mutation did not skew hematopoietic cells towards a tumor-promoting role, nor did it increase the mast cell abundance within the gastric tumor microenvironment.

Macrophages are reduced in gastric tumors in a mast celldependent manner. Because we observed mast cells outside of the tumor cores, we surmised that tumor-promoting effect of mast cells might occur indirectly by affecting the composition and/or function of tumor infiltrating immune cells. We therefore enumerated tumor-free and tumor-containing stomach sections collected from $g p 130^{\mathrm{FF}}$ and mast cell-deficient $g p 130^{\mathrm{FF}} ; c-K i t^{\mathrm{W}}$-sh/W-sh mice for the F4/80 macrophage, CD3 T lymphocyte and B220 B-lymphocyte markers. While we observed a $30 \%$ reduction in macrophage frequency in the submucosa and cores of tumors from $g p 130^{\mathrm{FF}} ; c$ $K i t^{\mathrm{W}-\mathrm{sh} / \mathrm{W} \text {-sh }}$ mice, the lymphocyte $\left(\mathrm{CD}^{+}\right)$, Treg $\left(\right.$Foxp3 $\left.3^{+}\right)$, and $\mathrm{CD}^{+} \mathrm{T}$ cell frequencies remained unchanged (Fig. 3a, b). Reduced macrophage abundance within the tumor core and submucosa was also observed in the mast cell-deficient $g p 130^{F F}$; Cpa3-Cre; Mcl1 fl/fl mice (Fig. 3c) and cromolyn-treated $g p 130^{\mathrm{FF}}$ mice (Supplementary Fig. 2g). Because genetic or pharmacological interference with mast cells not only inhibited tumor growth but concomitantly also reduced the abundance of tumor-associated macrophages, we postulate that a hierarchical mast cell-macrophage relationship underpins the growth of gastric cancer.

Macrophage infiltration coincides with gastric tumorigenesis. To ascertain that the accumulation of tumor-associated macrophages was a common occurrence for intestinal-type gastric cancers, we assessed macrophages density in the submucosa and mucosa of tumor-bearing $g p 130^{\mathrm{FF}}$ and $\mathrm{Tg}(T f f 1-C r e E R T 2) ;$ Pik3$c a^{\mathrm{H} 1047 \mathrm{R} /+}$; Pten ${ }^{\mathrm{fl} / \mathrm{fl}}$ mice. We noted an increased abundance of macrophages in the submucosa and the mucosa of either model (Fig. 3d, e and Supplementary 3a, b), consistent with the increased abundance of submucosal mast cells in both models (Fig. 1a, b and Supplementary Fig. 1a, b). To establish whether the increased macrophage accumulation occurs prior to tumor formation, akin to our observations with mast cells, we analyzed naïve $g p 130^{\mathrm{FF}}$ mice at 4 weeks of age. In these mice macrophage numbers were increased in the submucosal layers, but remained similar between the precancerous mucosa of $g p 130^{\mathrm{FF}}$ and of WT mice (Supplementary Fig. 3c, d). Collectively, these findings positively correlate macrophage numbers with gastric tumor growth and suggest a potential macrophage contribution to tumor formation and progression.

Macrophages are required for gastric tumorigenesis in $g p 130^{\mathrm{FF}}$ mice. Macrophages have been established as drivers of tumor growth and cancer therapy targets for several tumor entities 47,48 . To formally investigate a functional contribution of macrophages to gastric tumorigenesis, we generated macrophage-deficient $g p 130^{\mathrm{FF}} ; \mathrm{Csf1r^{-1- }}$ mice, because expression of the Csf1r gene and its corresponding receptor protein for macrophage colonystimulating factor is required for maturation of bone marrowderived macrophages (BMDM) from their monocytic precursors. Strikingly, the few surviving $g p 130^{\mathrm{FF}} ; \mathrm{Csf1r^{-1- }}$ mice remained completely free of gastric tumors (Fig. $4 \mathrm{a}, \mathrm{b}$ ). The submucosal mast cell density was not altered in the macrophage-deficient animals (Fig. 4c). We next confirmed that this phenotype reversion was not related to the runted phenotype of $g p 130^{\mathrm{FF}} ; \mathrm{Cs} f 1 r^{-1-}$ mice associated with their feeding requirement for mashed chow to overcome the Csf1R deficiency-dependent absence of teeth. We therefore depleted macrophages in adult tumor-bearing $g p 130^{\mathrm{FF}}$ mice by systemic administration of clodronate-loaded liposomes (clodrosomes) for 6 consecutive weeks and observed a significant reduction of tumor burden when compared to the vehicle control cohort (Fig. 4d). Although toluidine-staining confirmed that clodrosome treatment did not reduce the abundance of submucosal mast cells, gastric tumors and adjacent submucosa from the clodrosome cohort revealed reduced density of CD31-positive microvessels (Fig. 4e and Supplementary Fig. 4a).

The reduced tumor-burden and microvessel density observed in the clodronate cohort were similar to our findings from the mast cell depletion experiments. We next aimed to exclude that they relate to off-target-effects associated with bisphosphonate (i.e., clodronate) administration. We therefore treated tumorbearing $g p 130^{\mathrm{FF}}$ mice with the small molecule Csfr1/c-kit/Flt3 kinase inhibitor PLX3397 (Pexidartinib). We supplied PLX3397 in the food to tumor-bearing $g p 130^{\mathrm{FF}}$ mice for 4 weeks (acute therapeutic) and continued a cohort for a further 4 weeks without PLX3397 treatment (follow-up) (Supplementary Fig. 4b). Acute PLX3397 treatment revealed a significant reduction in tumor burden, although this antitumoral effect was reversible as the tumor burden increased once PLX3397 treatment ceased in the follow-up cohort (Fig. 5a). In the acute treatment cohort, we confirmed by immunohistochemical staining a significant reduction of F4/80-positive macrophages accompanied by reduced density of CD31-positive angiogenic vessels and increased staining for tumor-hypoxia (Fig. 5b and Supplementary Fig. 4c). However, owing to the specificity of PLX3397, we also observed a decrease of tumor-adjacent submucosal mast cells. All these parameters reverted back to their pretreatment state following a drug-free period in the follow-up cohort (Supplementary Fig. 4c).

Based on our proposed role for macrophages to mediate the tumor promoting effect of mast cells, we hypothesized that the tumor-associated macrophages in the gastric lesions of $g p 130^{\mathrm{FF}}$ mice should have a tumor-promoting alternatively activated endotype. We therefore compared expression of macrophage endotype markers between naïve BMDM and tumor-associated macrophages, and used standard in vitro polarization protocols for BMDM to generate a reference population for alternative activation. For the tumor-associated macrophage populations, this analysis revealed high expression of the alternative activation markers Arg1, Fizz1, and Mrc1, which coincided with reduced mRNA levels of the classically activated marker Nos2 (Fig. 5c). Consistent with the pronounced presence of microvasculature in tumors of these $g p 130^{\mathrm{FF}}$ mice, we also recorded elevated expression of angiogenic Vegfa in the tumor-associated 


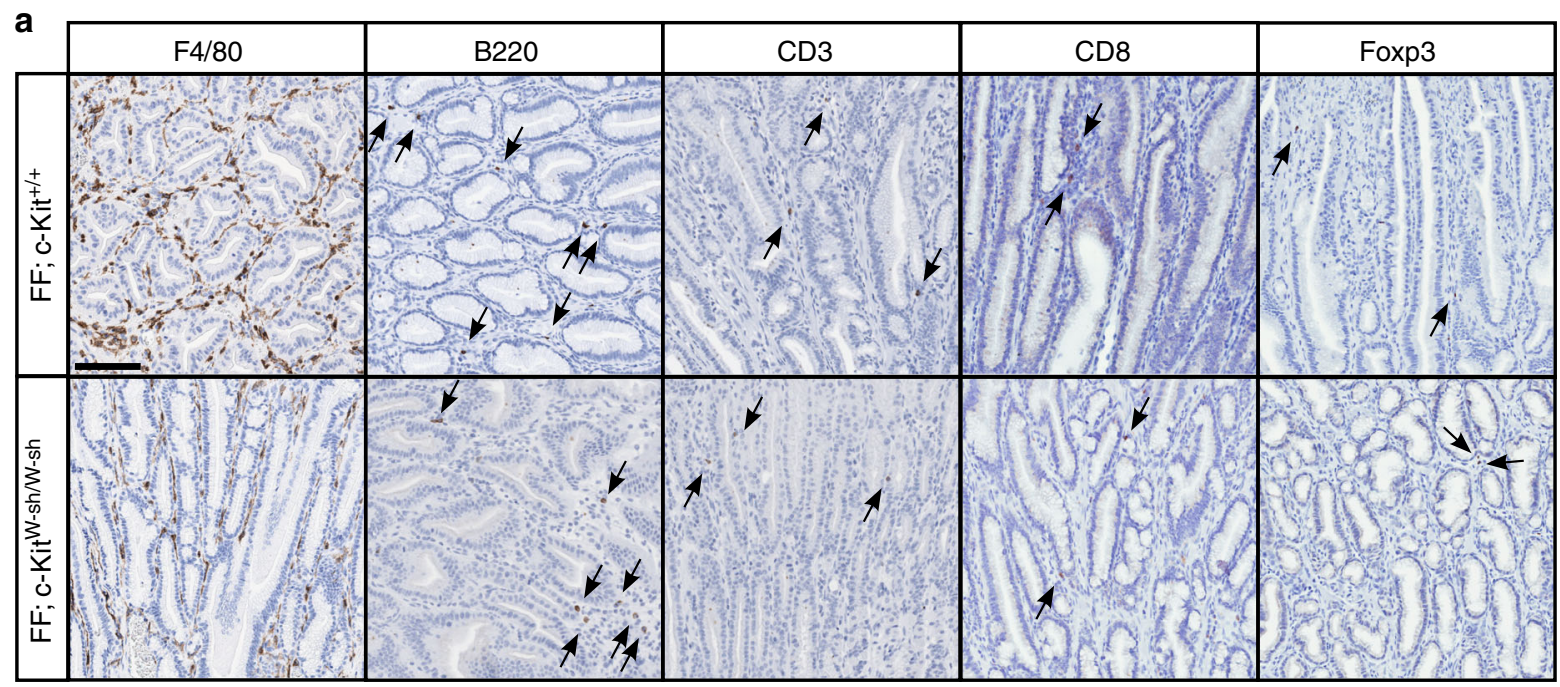

b $\quad \mathrm{FF} ; \mathrm{c}-\mathrm{Kit}^{+/+} \square \mathrm{FF} ; \mathrm{c}-\mathrm{Kit}{ }^{\mathrm{W}-\mathrm{sh} / \mathrm{W}-\mathrm{sh}}$
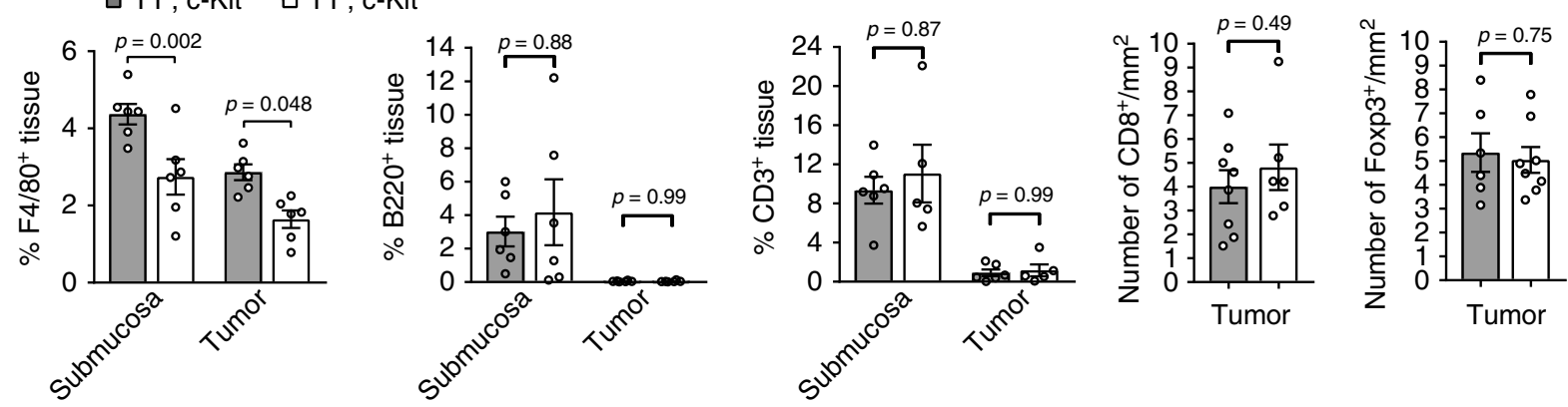

C

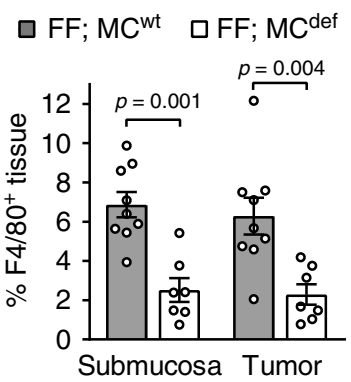

d

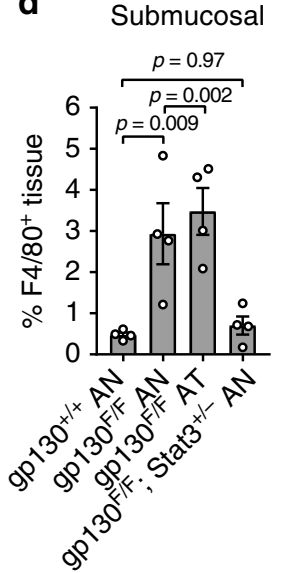

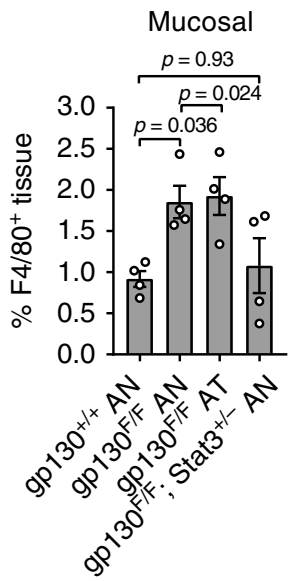

e

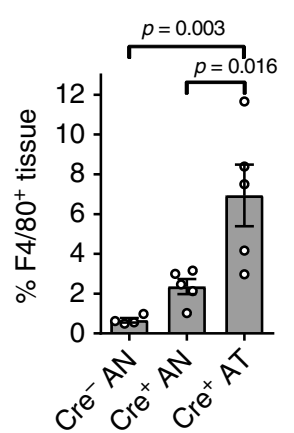

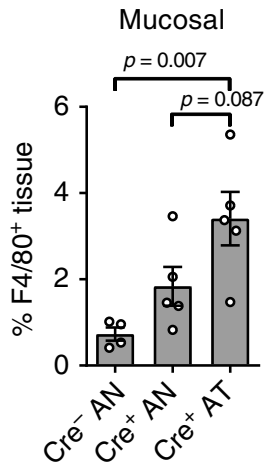

Fig. 3 Mast cell depletion reduces macrophage infiltration in gastric tumors of gp130 $\mathrm{FF}$ mice. a Representative images of tissue sections immunostained for macrophages (F4/80), B cells (B220) or T cells (CD3, CD8, Foxp3) in gastric tumor sections of gp130FF; c-Kit $+/+$ and mast cell-deficient $g p 130^{F F}$; c-KitWsh/W-sh mice. Arrows indicate specific positive cell staining; scale bars $=100 \mu \mathrm{m}$. b Quantification of F4/80, B220, or CD3 expressing cells in tumor and tumor-associated submucosal layers of sections from (a). F4/80: $n=6$ each group, one-way ANOVA F (DFn, Dfd) = 13.43 (3, 20); B220: $n=6$ each group, one-way ANOVA F (DFn, Dfd) $=3.782(3,20)$; CD3: FF; c-Kit ${ }^{+/+} n=6, F F ; c-K i t W-s h / W$-sh $n=5$, one-way ANOVA F (DFn, Dfd) = 11.55 (3, 18); CD8: FF, c-Kit ${ }^{+/+} n=8, F F ; c-K i t^{W-s h / W-s h} n=6, t$-test t (df) $=0.71$ (12); Foxp3: FF, c-Kit ${ }^{+/+} n=6, F F ; c-K i t^{W}$-sh/W-sh $n=8, t$-test t (df) $=0.33$ (12). Data from two independent experiments were analyzed. c F4/80+ cells frequency in stomach submucosa and tumors of $F F$; $M C^{w t}$ mice $(n=9)$ and mast celldeficient FF; MCdef mice $(n=7$ FF;Cpa3-Cre; Mclffl/fl). One-way ANOVA F (DFn, Dfd) $=10.81(3,28)$. d Quantification of F4/80+ cells in unaffected antrum (AN) or antrum tumor (AT) in submucosa or mucosa of mice of the indicated genotype. All groups $n=4$. Submucosal: one-way ANOVA $F(D F n, D f d)=$ $10.12(3,12)$; Mucosal: one-way ANOVA F (DFn, Dfd) $=5.086(3,12)$. e Quantification of F4/80+ cells in unaffected antrum (AN) or antrum tumors (AT) in submucosa or mucosa of mice from the Tg(Tff1-CreERT2); Pik3ca H1047R/+; Pten fl/fl strain that either harbor (Cre ${ }^{+}$) or lack (Cre ${ }^{-}$) the Tff1-CreERT2 driver. $\mathrm{Cre}^{-} \mathrm{AN} n=4, \mathrm{Cre}^{+} \mathrm{AN}$ and Cre ${ }^{+} \mathrm{AT} n=5$ for both. Analyses from two independent experiments. Submucosal: one-way ANOVA F (DFn, Dfd) $=10.52$ (2, 11); Mucosal: one-way ANOVA F (DFn, Dfd) $=7.485(2,11)$. Data are represented as mean \pm SEM, with $p$ values $p<0.05$ considered being significant. Source data are provided as a Source Data file. See also related Supplementary Fig. 3 

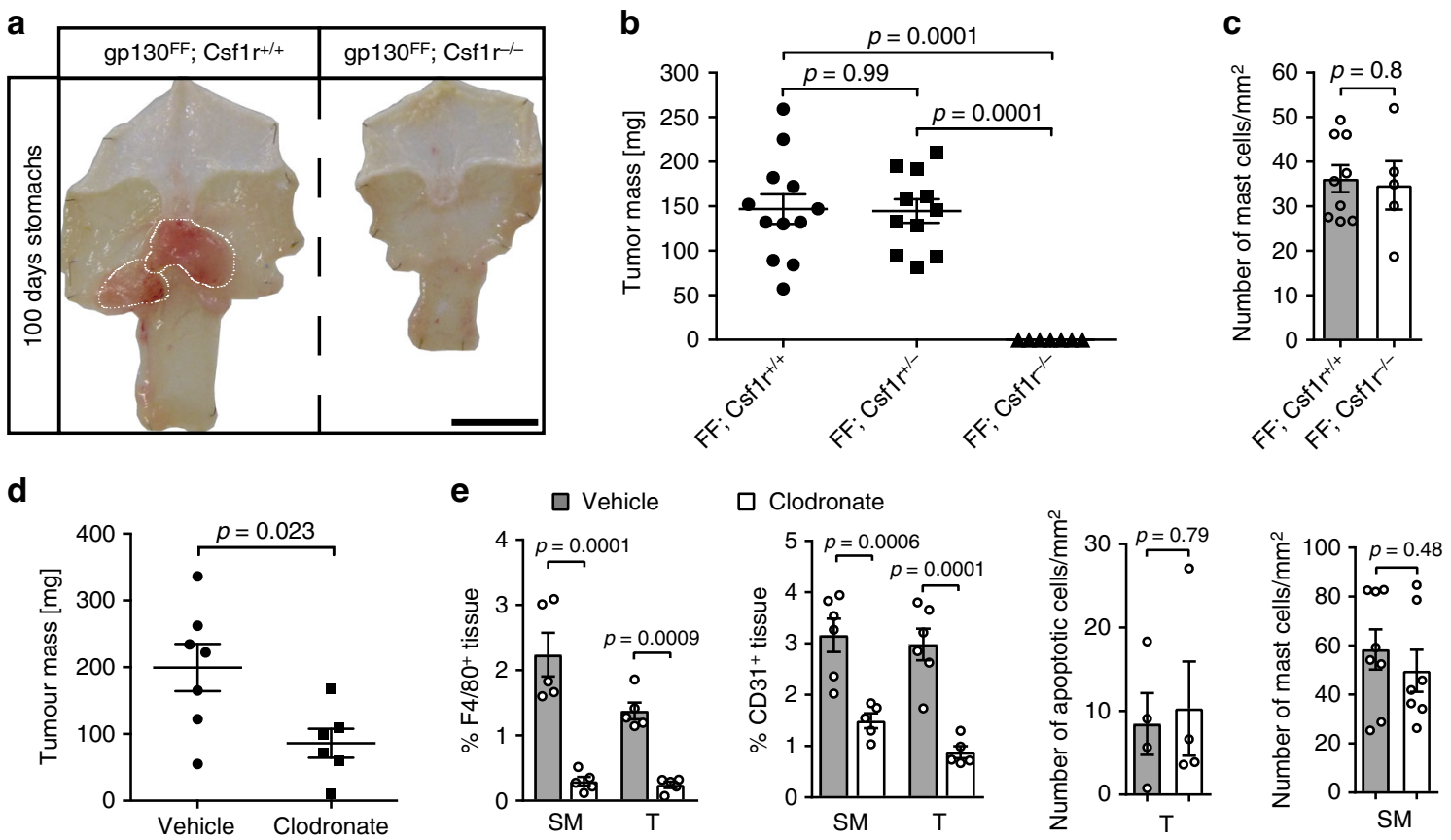

Fig. 4 Tumor burden in gp130 FF mice diminishes upon macrophage depletion. a Representative whole mount of stomachs from 100 -day-old mice of the indicated genotype. Scale bar $=1 \mathrm{~mm}$. b Quantification of total tumor burden per mouse as in (a). Each symbol represents an individual mouse. Due to ill health some gp130F/F; Csf1r-/- mice had to be analyzed before the 100 day endpoint; average mouse age per groups were: gp130F/F. Csf1r+/+ 88.2 days, gp130 F/F; Csf1r //- 90.4 days, and gp130F/F. Csf1r /- 81.6 days. One-way ANOVA F (DFn, Dfd) $=27.55$ (2, 27 ). c Quantification of mast cell density in antral submucosa of indicated genotype was performed with gp130F/F; Csf1r $+/+n=9$ and gp $130 \mathrm{~F} / \mathrm{F}$; Csf1r $-/-n=5$ biological samples from two independent experiments $t$-test $\mathrm{t}(\mathrm{df})=0.262$ (12). d Assessment of total tumor burden in gp130 FF mice after 6 weeks administration of clodronate ( $50 \mu \mathrm{l}$ of a clodrosome solution containing $5 \mathrm{mg} / \mathrm{ml}$ clodronate) or vehicle. Each symbol represents an individual mouse; data from two independent experiments ( $t$-test $t(d f)=2.622(11)$ ). e Quantification of F4/80, CD31, ApopTag, or toluidine blue (for detection of mast cells) stained sections of gastric tumors ( $T$ ) and tumor-associated submucosa (SM) from gp130 FF mice of the indicated treatment cohort. F4/80: all $n=5$, one-way ANOVA F (DFn, Dfd) = 27.17 (3, 16); CD31: $n=6$ (SM tissues), $n=5$ (T tissues), one-way ANOVA F (DFn, Dfd) $=18.15$ (3, 18); apoptotic cells: $n=4$ (both groups), $t$-test $t$ (df) $=0.27$ (6); mast cells: $n=8$ (vehicle) and $n=7$ (Clodronate), $t$-test $t(\mathrm{df})=0.73$ (13). Data are represented as mean \pm SEM, with $p$ values $p<0.05$ considered being significant. Source data are provided as a Source Data file. See also related Supplementary Fig. 4

macrophage population when compared to the naïve BMDM population from the same mice (Fig. 5c). Given the alternative activated endotype of tumor-associated macrophages, our data collectively suggests that their abundance is likely to be a ratelimiting factor for the establishment of tumor microvasculature and the growth of gastric tumors.

Tumor-derived IL-33 activates mast cell secretion and expression of macrophage-attracting chemokines. Although our observations thus far suggested a functional mast cell-macrophage relationship, it remained to determine the molecular network underpinning this hierarchy including how its activation could occur. We therefore focused on IL-33 as a potential tumor-derived activator of mast cells, because IL-33 was reported to serve as an alarmin that is generated and released by necrotic cells, and that triggers mast cell degranulation ${ }^{49}$. Indeed, we detected increased expression of $\mathrm{Il} 33$ in the EpCam ${ }^{+} / \mathrm{CD}_{4} 5^{-}$ epithelial components of the tumors in $g p 130^{\mathrm{FF}}$ mice with a reciprocal expression of the Il1rl1 gene in the hematopoietic $\mathrm{CD} 5^{+} / \mathrm{EpCam}^{-}$compartment of the antrum, which encodes the cognate St2 receptor subunit for IL-33 (Fig. 6a). In line with the reported storage of IL-33 protein in vesicles, we detected increased levels of Il33 mRNA and IL-33 protein in whole tumor tissue (Supplementary Figs. 5a, b). Immunofluorescence staining revealed that IL-33 protein localized most prominently to the peripheral regions of the epithelial components of the tumors (Fig. 6b) coinciding with the location of the highest level of cellular turn-over ${ }^{37}$.
To assess whether tumor-associated mast cells could respond to IL-33, we FACS purified the $\mathrm{c}-\mathrm{Kit}^{+} / \mathrm{F}_{c} \varepsilon \mathrm{R} 1^{+}$mast cells from the submucosal antrum of WT and $g p 130^{\mathrm{FF}}$ mice using stomachs from $g p 130^{\mathrm{FF}} ; c-K i t^{\mathrm{W}-\mathrm{sh} / \mathrm{W} \text {-sh }}$ mice as a specificity control for our purification strategy (Supplementary Fig. 5c). We confirmed the homogeneity of the $\mathrm{c}-\mathrm{Kit}^{+} / \mathrm{F}_{\mathrm{c}} \varepsilon \mathrm{R} 1^{+}$mast cell population by toluidine blue staining (Supplementary Fig. 5d) and showed prominent expression of the St2 receptor in the $\mathrm{c}-\mathrm{Kit}^{+} / \mathrm{F}_{\mathrm{c}} \varepsilon \mathrm{R} 1^{+}$ mast cells isolated from the stomach of $g p 130^{\mathrm{FF}}$ mice (Supplementary Fig. 5e). We then stimulated this cell population for $3 \mathrm{~h}$ with IL-33 and detected by the multiplex cytokine assay increased amounts of IL-6 and IL-13 and to lesser extent of IL-1a, IL-4, alongside elevated secretion of the macrophage growth and attracting factors Gm-csf, Mcp1, Mip-1 $\alpha$, and Mip-1 $\beta$ (Supplementary Fig. 5f and Fig. 6c). Likewise, qPCR analysis of the IL-33 stimulated mast cells also revealed increased expression of $\mathrm{Ccl} 2$ (encoding Mcp1 protein), Ccl3 (encoding Mip-1 $\alpha$ protein), and Ccl7 (Fig. 6d), which collectively exert chemotactic activity towards macrophages and neutrophils ${ }^{50-52}$. Also, IL-33stimulation induced elevated expression of angiogenic growth factor gene Vegfa (Fig. 6d). In order to confirm the chemotactic activity of the mast cell produced factors on $g p 130^{F F}$ macrophages, we performed migration assays with BMDM. Indeed, Mcp1 and Mip-1a alone induced migration significantly (Supplementary Fig. 5g).

Because $g p 130^{\mathrm{FF}}$-driven gastric tumorigenesis has an absolute requirement for IL-11 signaling $^{38}$, we predicted that IL-11 could stimulate IL-33 expression in tumor-derived epithelium. To test 
a

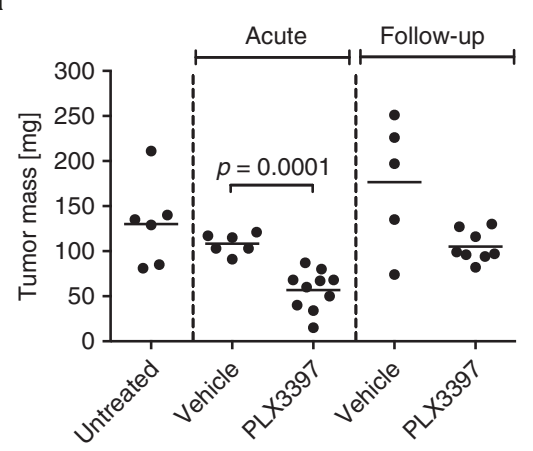

b

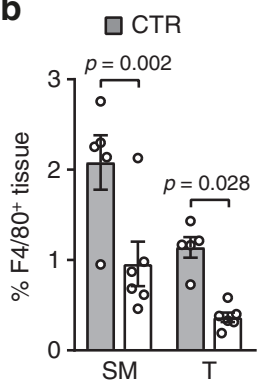

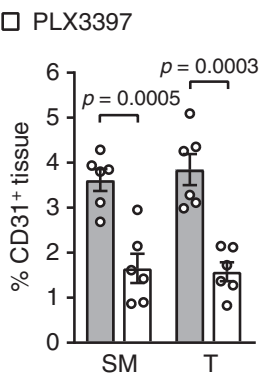
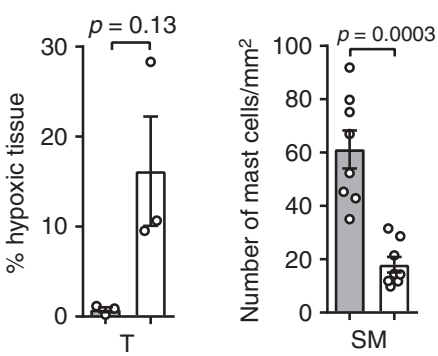
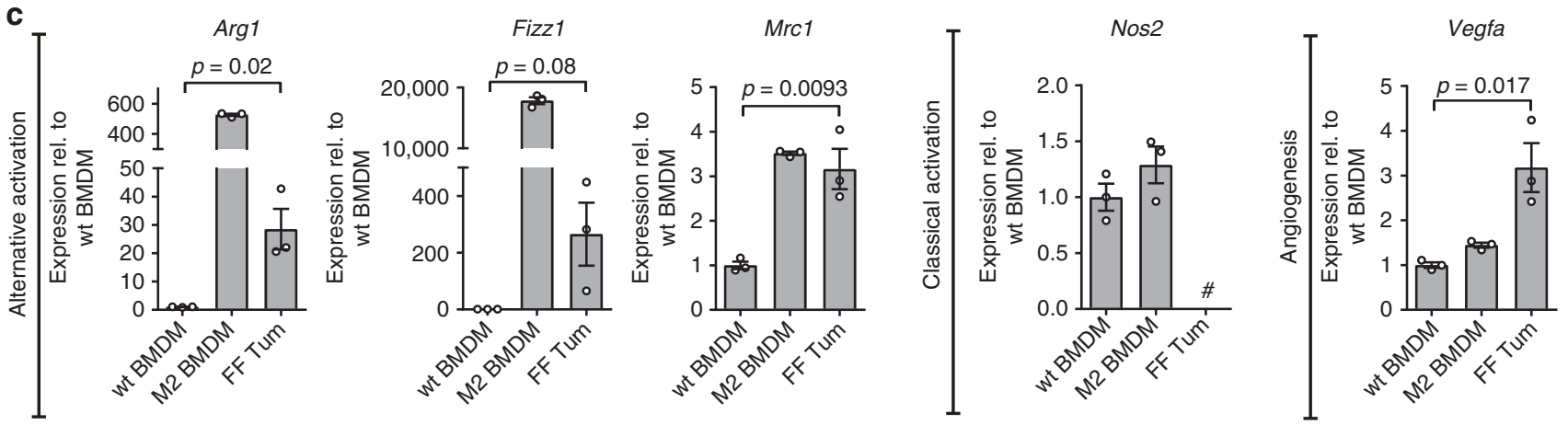

Fig. 5 Pharmacological macrophage targeting and macrophage polarization in gp130 FF tumors. a Total tumor burden in gp130 $\mathrm{FF}$ mice was quantified either acutely after a 4-week-treatment period with PLX3397 (Csf1r/c-Kit/Flt3 tyrosine kinase receptor inhibitor) or vehicle or after a 4 weeks treatment-free follow-up period. Each symbol represents an individual mouse. Data from three independent experiments are presented. $t$-test $t(d f)=5.23(14)$. b Quantification of F4/80, CD31, Hypoxyprobe or toluidine-blue-stained sections of submucosa (SM) or gastric tumors (T) of gp130 FF mice of the indicated acute treatment cohort. F4/80: $n=5$ (Vehicles) and $n=6$ (PLX3397), one-way ANOVA F (DFn, Dfd) $=12.34$ (3, 18); CD31: $n=6$, one-way ANOVA F $(\mathrm{DFn}, \mathrm{Dfd})=18.33(3,20)$; hypoxic tissue: $n=3$, $t$-test with Welch's correction $\mathrm{t}(\mathrm{df})=2.53$ (2); mast cells: $n=8$ (both groups), and $t$-test with Welch's correction $t(\mathrm{df})=5.59$ (9.27). c qPCR expression analysis of genes associated with alternative activation (Arg1, Fizz1, Mrc1), classical activated (Nos2) and angiogenesis (Vegfa) of purified F4/80+ tumor-associated macrophages (FF Tum) or following stimulation of bone marrow-derived macrophages (BMDM) from wild-type mice and stimulated either with vehicle (wt BMDM) or with IL-4/IL-13 (20 ng/ml) to induce an alternative activated endotype (M2 BMDM). $n=3$ mice. Hash indicates that expression was below detection limit. Arg1: $t$-test $t(d f)=3.8$ (4); Fizz1: $t$-test $t$ (df) $=2.39$ (4); Mrc1: $t$-test $t$ $(d f)=4.69$ (4); Vegfa: $t$-test $t(d f)=3.96$ (4). Data are represented as mean $\pm S E M$, with $p$ values $p<0.05$ considered being significant. Source data are provided as a Source Data file. See also related Supplementary Fig. 4

this, we exposed epithelial organoids grown from $g p 130^{F F}$-tumors to recombinant IL-11 in vitro. While organoid growth and morphology were not altered (Fig. 6e and Supplementary Fig. 5h), we observed a marked increase in Il33 expression upon IL-11 stimulation, which was comparable to the extent of induction of the bona fide IL-11/Stat3-target gene Socs3 (Fig. 6f). Additionally, we conducted a chromatin immunoprecipitation (ChIP) experiment on DNA from gastric tumors of $g p 130^{\mathrm{FF}}$ mice 60 min after acute systemic administration of IL-11. The ChIP experiment revealed a Stat3-specific binding peak on chromosome 19, which maps to the $5^{\prime}$-region of the Il33 gene and which contains a Stat3binding site motif (Supplementary Fig. 5i, j). Collectively, these data indicate that tumor-derived IL-11 induces Il33 gene expression in gastric tumor cells, which triggers the secretion as well as the de novo synthesis of cytokines and chemokines by mast cells to produce a tumorigenic immune environment.

IL-33/St2 signaling deficiency decreases $g p 130^{\mathrm{FF}}$-mediated gastric tumor growth. To validate a functional contribution of IL33 signaling as a master regulator of mast cell activation in the gastric tumor microenvironment, we generated IL-33 signaling deficient $g p 130^{\mathrm{FF}} ;$ St2 $2^{-1-}$ mice. Compared to their St2 ${ }^{+1+}$ and $S t 2^{+l-}$-proficient compound mutant littermates, $g p 130^{\mathrm{FF}}$; St $2^{-1-}$ mice harbored a significantly lower overall tumor burden at 100 days of age (Fig. 7a), which arose from reduced tumor growth rather than reduced tumor incidence (Fig. 7b). These observations coincided with a decrease in mast cell numbers in the tumoradjacent submucosal layers and reduced density of $\mathrm{F} 4 / 80^{+}$macrophages in the tumors of $g p 130^{\mathrm{FF}} ; \mathrm{St} 2^{-1-}$ mice as well as reduced abundance of CD31-positive microvessels (Fig. 7c).

We next confirmed that $\mathrm{c}-\mathrm{Kit}^{+} / \mathrm{F}_{\mathrm{c}} \varepsilon \mathrm{R} 1^{+}$mast cells isolated from $g p 130^{\mathrm{FF}} ; \mathrm{St}^{-1-}$ mice showed significantly decreased expression of the macrophage recruiting and inflammatory mediators Csf2 (encoding Gm-csf), Ccl3, and Il6 (Fig. 7d). Because IL-33 signaling is involved in expansion and activation of ILC2 and Tregs, which could also afford a mechanism for IL-33 to promote gastric tumorigenesis in $g p 130^{\mathrm{FF}}$ mice, we assessed the relative abundance of ILC2 and Tregs among all cells harboring the panhematopoietic surface marker CD45. We observed comparable frequency of these cells between unaffected antrum and tumors of $g p 130^{\mathrm{FF}}$ and $g p 130^{\mathrm{FF}} ;$ St2 ${ }^{-1-}$ mice. Moreover, less than $30 \%$ of all tumor-associated ILC2 cells, and less than 15\% of all Tregs, expressed the St 2 receptor (Fig. 7e, f).

To investigate the involvement of mast cells in the antitumoral effect of St2-deficiency, we established bone marrow-derived mast cells (BMMC) from $g p 130^{F F} ; S T 2^{-/-}$and $g p 130^{F F}$; St2 ${ }^{+/+}$mice, confirmed their purity (Supplementary Figs. 6a-c) and performed adaptive transfer of BMMC into $g p 130^{F F}$; St2-l- hosts (Supplementary Fig. 6d). Reconstitution with $\mathrm{St}^{+}$wild-type BMMC $\left(g p 130^{F F} ; S_{2}{ }^{+/+}\right)$increased tumor burden compared to littermates injected with $g p 130^{F F}$; St2 ${ }^{-/-}$BMMCs (Fig. $7 \mathrm{~g}$ and Supplementary 
a

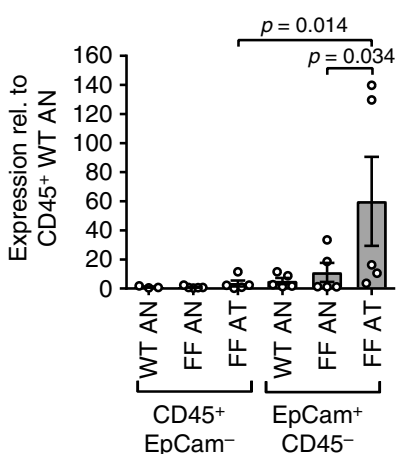

$\| 1 r / 1$

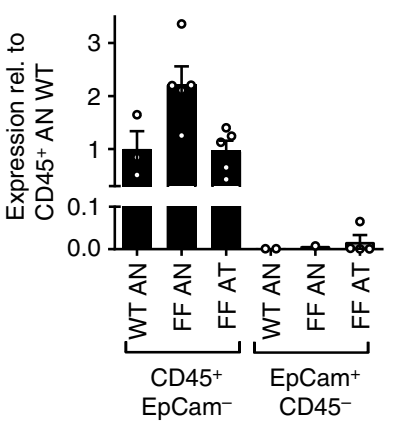

b

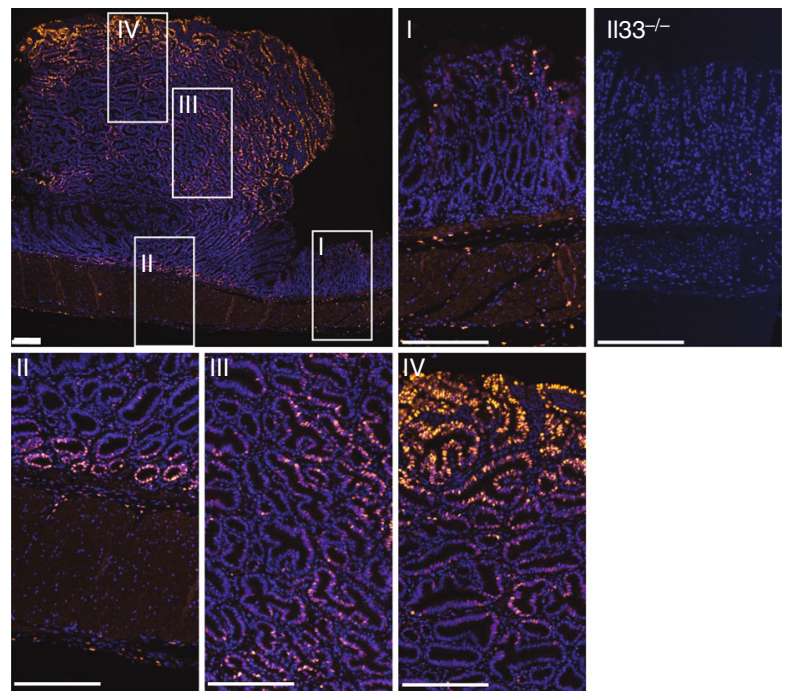

d
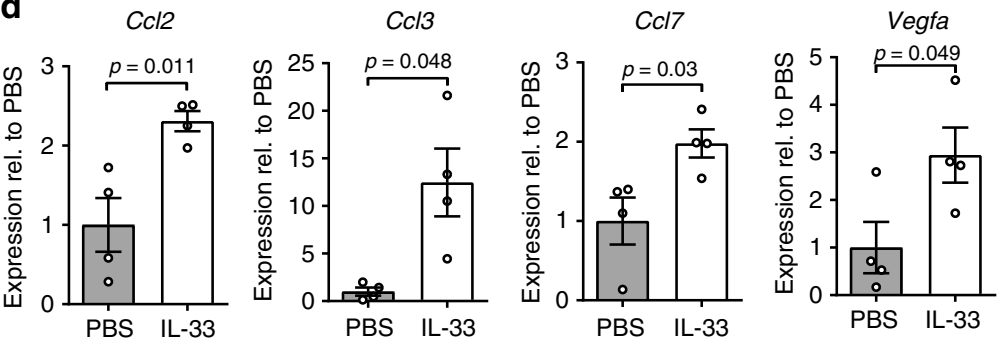

e

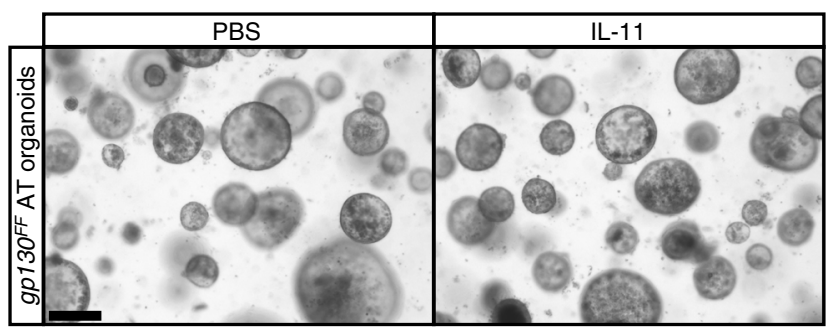

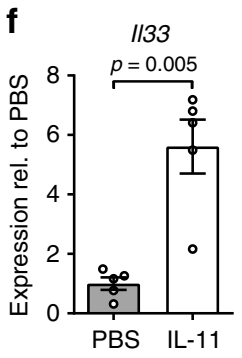

Fig. 6 IL-33 expression in gastric tumors of gp130FF mice and mast cell activation analysis. a qPCR expression analysis of II33 and full-length St2 (I/1r/1) genes associated with hematopoietic ( $\left.\mathrm{CD} 45^{+} ; \mathrm{EpCam}^{-}\right)$and epithelial $\left(\mathrm{EpCam}^{+} ; \mathrm{CD} 45^{-}\right)$cells purified from unaffected antrum (AN) or antrum tumors (AT) from wild-type and gp $130^{\mathrm{FF}}$ (FF) mice. Data are normalized to Gapdh and plotted as relative expression to CD45+; EpCAM- WT AN expression. $n=5$ mice. IIrl1 expression in EpCam ${ }^{+}$; CD45- cells was for several samples below detection limit. Data was pooled from two independent experiments. For IL33 data, one-way ANOVA was performed with F (DFn, Dfd) $=2.871(5,22)$; b Immunofluorescence staining for IL-33 in stomachs of tumor-bearing gp130 FF mice with insets referring to unaffected antrum (I), submucosal-tumor junction (II), tumor core (III), and tumor edge (IV). Stomachs from II33-/mice were used for specificity controls. Scale bars $=200 \mu \mathrm{m}$. c Multiplex cytokine analysis of supernatant of FACS-purified tumor-associated mast cells stimulated with IL-33 (30 ng/ml) for $3 \mathrm{~h}$. Data are shown only for factors with $>3$ fold increase relative to unstimulated control cultures. $n=4$ from four independent experiments. d qPCR gene expression analysis in FACS-purified tumor-associated mast cells stimulated with IL-33 (30 $\mathrm{ng} / \mathrm{ml}$ ) for $3 \mathrm{~h}$ or

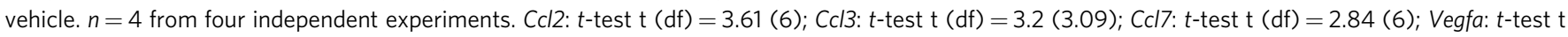
$(\mathrm{df})=2.45$ (6). e Representative images of organoids derived from antral tumors (AT) of gp130 FF mice stimulated either with PBS or IL-11 (100 ng/ml for 2 days). Scale bar $=200 \mu \mathrm{m}$. $\mathbf{f} / 133$ gene expression analysis of $g p 130^{\mathrm{FF}}$ tumor-derived epithelial organoids either stimulated with IL-11 (100 $\mathrm{ng} / \mathrm{ml}$ ) or with PBS for $4 \mathrm{~h}$. For comparison expression of Stat3-target gene, Socs3, was also analyzed. $n=5$ from two independent experiments. Stat3: $t$-test $t$ (df) $=4.96$ (4.42); Socs3: $t$-test $t(d f)=5.6$ (4.48). Data are represented as mean $\pm S E M$, with $p$ values $p<0.05$ considered being significant. Source data are provided as a Source Data file. See also related Supplementary Fig. 5

Fig. 6e). At the time point of tumor analysis $8 \%$ of the gastric submucosal mast cells stained positive for St 2 expression and therefore represent the transplanted BMMC mast cells, which homed to the gastric tumor site and compete with the endogenous ST2 ${ }^{-1-}$ mast cell compartment (Supplementary Fig. 6f).

These observations further support a mechanism by which IL33 promotes gastric tumorigenesis through mast cell activation rather than by promoting ILC2 or Treg accumulation.
IL-33/mast cells-activity gene expression signature predicts poor survival for human intestinal-type gastric cancer. In order to translate our preclinical findings in mice to a relevant setting in humans, we hypothesized that the extent of mast cell activation might provide retrospective prediction of patient survival. Based on the mast cell activation gene expression signature derived from our in vitro mast cell stimulation with IL-33, we analyzed a publically available data set (DErrico Gastric data set; GEO: 
a

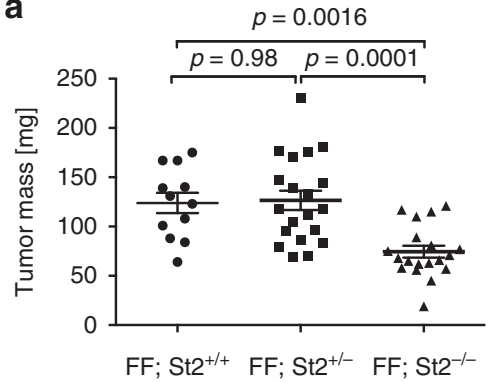

b

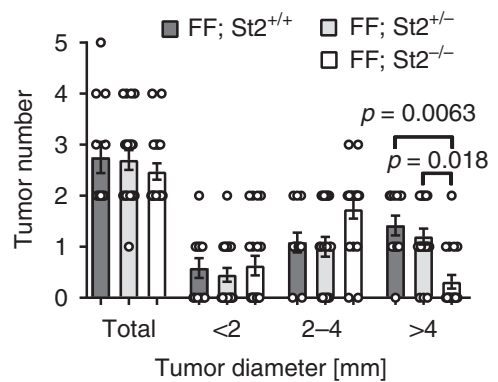

C
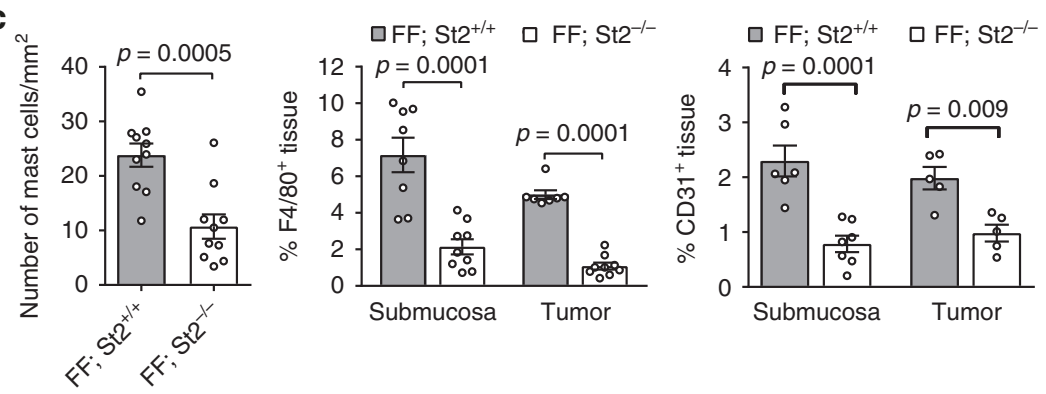

d
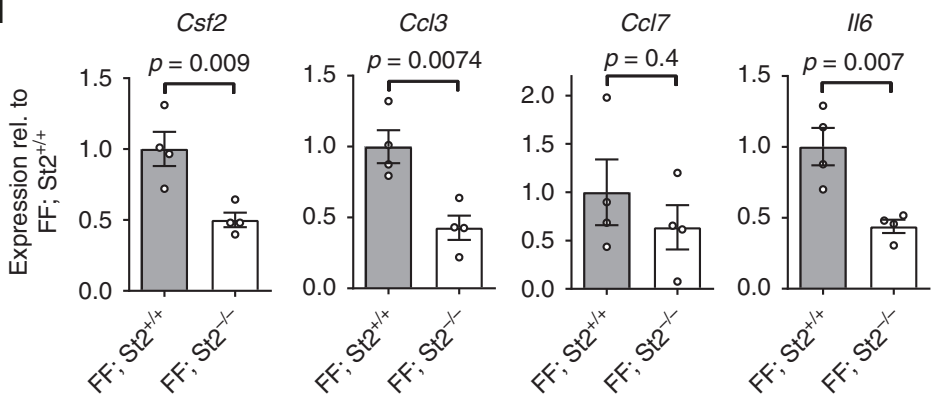

e
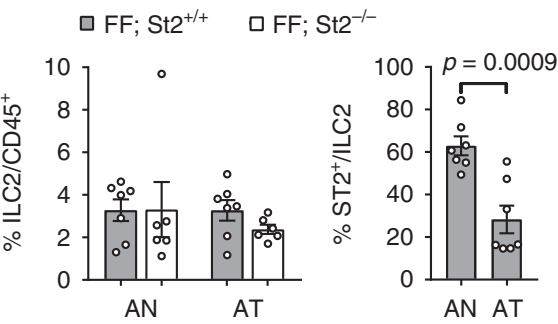

f
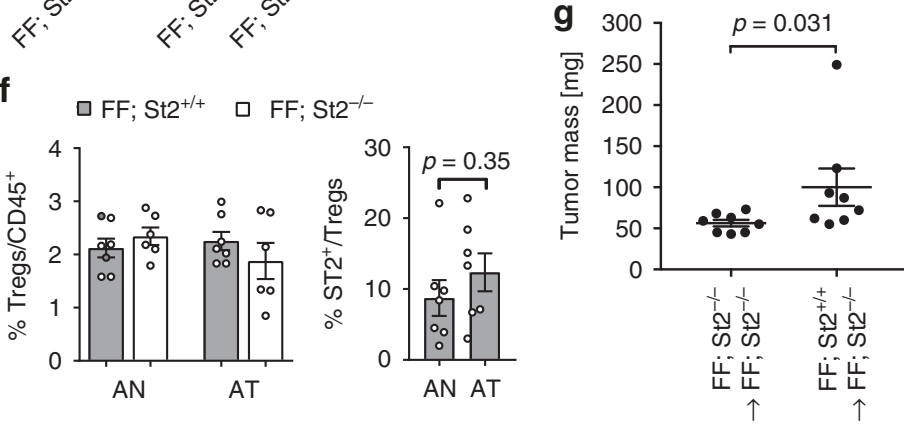

Fig. 7 Tumor burden is reduced in St2 receptor-deficient gp130 FF mice. a Quantification of total tumor burden in 100-day-old mice of the indicated genotype. Each symbol represents an individual mouse. One-way ANOVA was performed with $F(D F n, D f d)=11.83(2,48)$. $\mathbf{b}$ Enumeration of total tumor number from mice in $\mathbf{a}$, and of tumors following classification according to their size. $n=12\left(F F, S t 2^{+/+}\right), n=20\left(F F\right.$, St2 $\left.2^{+/-}\right)$, and $n=19\left(F F, S t 2^{-/-}\right)$mice. One-way ANOVA was performed with $F(D F n, D f d)=22.79(11,192)$. c Quantification of toluidine blue (for detection of mast cells; submucosal tissue), F4/80 and CD31 stained sections of gastric tumors of mice of the indicated genotype. Mast cells: $n=10$ mice, $t$-test $t$ (df) $=4.25$ (18); F4/80: $n=8$ (FF; St2 $\left.{ }^{+/+}\right), n=9\left(\mathrm{FF} ; \mathrm{St}^{-/-}\right.$), one-way ANOVA F (DFn, Dfd) $=27.52(3,29) ; \mathrm{CD} 31: n=6$ (Submucosa) $n=5$ (Tumor), and one-way ANOVA F (DFn, Dfd) $=13.6(3,19)$. $\mathbf{d}$ qPCR expression analysis of chemokines expressed by FACS-purified tumor-associated mast cells from stomachs of either FF; St2 $+/+$ or $F F$; $S t 2^{-/-}$mice. All $n=4$ from two independent experiments. Csf2: $t$-test $\mathrm{t}(\mathrm{df})=3.81(6) ; C \mathrm{cl} 3: \mathrm{t}$-test $\mathrm{t}(\mathrm{df})=3.97(6) ; C \mathrm{cl} 7: t$-test $\mathrm{t}(\mathrm{df})=0.88(6) ; 116:: t$-test $t(d f)=4.02(6) ; \mathbf{e}$, f Flow cytometric analysis of unaffected antrum (AN) and antrum tumors (AT) of indicated genotype for the frequency of ILC2 cells

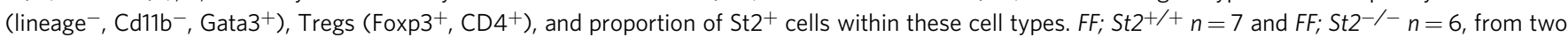
independent experiments. ST2 + ILC2: $t$-test $\mathrm{t}(\mathrm{df})=4.39(12)$; ST2 $+/$ Treg: $t$-test $\mathrm{t}(\mathrm{df})=0.98(12) . \mathbf{g}$ Enumeration of total tumor burden at 14 weeks of age of $F F$; St2 ${ }^{-/}$- host mice, which received tail vein injections of either FF; St2-/- or FF, St2 ${ }^{+/+}$bone marrow-derived mast cells (BMMC) ( $n=8$ mice per group). Mann-Whitney test was performed with Mann-Whitney $U=11.5$. Data are represented as mean \pm SEM, with $p$ values $p<0.05$ considered being significant. Source data are provided as a Source Data file. See also related Supplementary Fig. 6

GSE13911) for differential expression of the mast cell signature comprising CCL2, CCL3, CCL4, IL1a,IL4,IL6, IL13, CSF2, and CCL7. With the exception of IL-4 and IL-13, we found that all other factors were at least 1.6-fold increase in the cancer samples $(p \leq 0.05)$ (Fig. 8a). We therefore assigned the differentially expressed factors (i.e., CCL2, CCL3, CCL4, IL1a, IL6, CSF2, and CCL7) to an IL-33/mast cell activation gene expression signature for a Kaplan-Meier survival analysis (KMplot.com) ${ }^{53}$. 
a

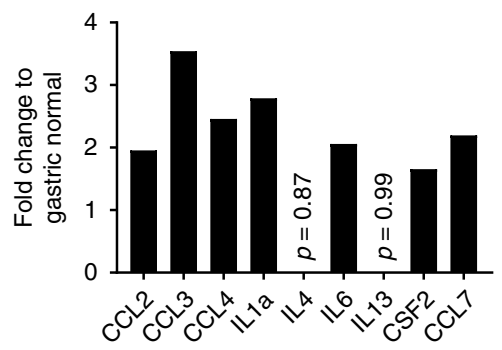

C

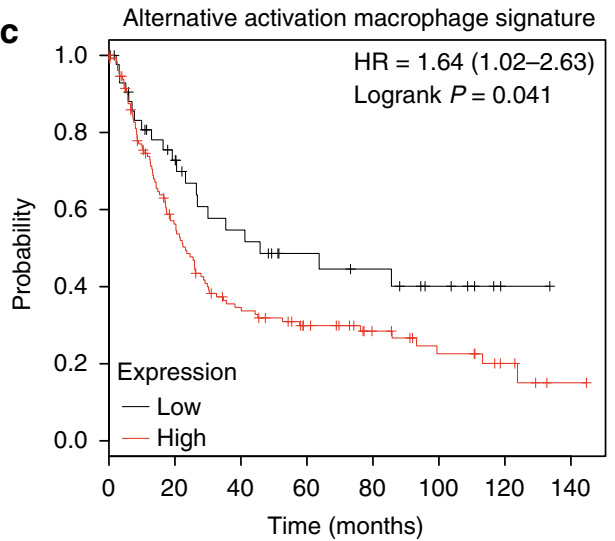

b

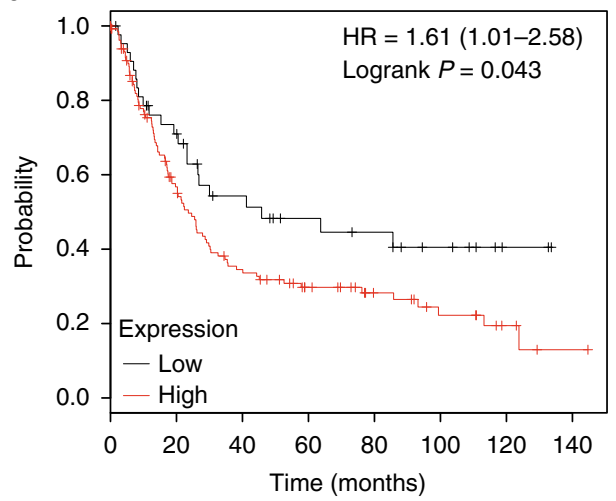

d Classical activation macrophage signature

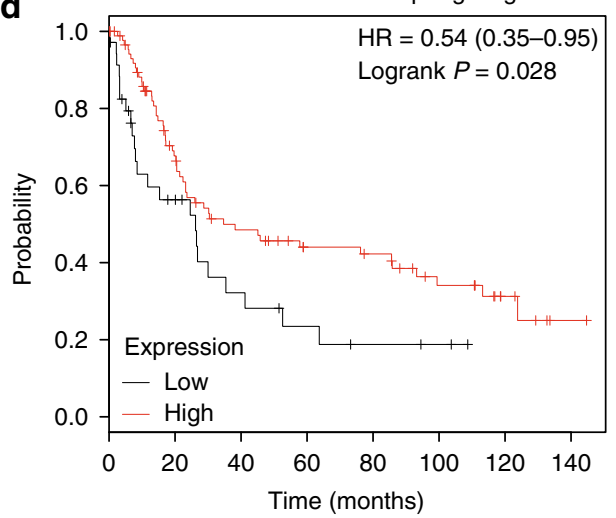

e

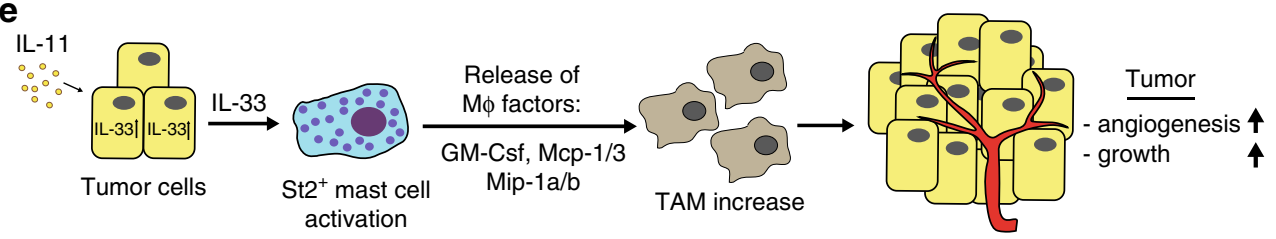

Fig. 8 Kaplan-Meier analysis for an IL-33 - mast cell activation gene expression signature. a Expression analysis of genes associated with IL-33 dependent mast cell activation derived from the DERRICO gastric data set (GEO: GSE13911, extracted from oncomine.org) and comparing normal gastric mucosa ( $n=$ 31) and gastric intestinal-type adenocarcinoma $(n=29)$. The $p$-value for the genes is $p<0.05$ except where indicated. b-d Kaplan-Meyer survival analysis was performed for human intestinal-type gastric cancer (iGC) with IL-33/ mast cell activation signature consisting of CCL2, CCL3, CCL4, IL7a, IL6, CSF2, and CCL7 (b), with alternative activation macrophage expression signature (CD163, CD204, MARCO, ARG1) (c) and classical activation macrophage expression signature (NOS2A, HLA-DRA, CD80, CD86, CD169) (d). e Schematic illustration of the proposed gastric cancer growth promoting IL-11/IL-33/mast cell/ TAM signaling axis. Source data are provided as a Source Data file. See also related Supplementary Fig. 7

Remarkably, we found a lower overall survival for intestinal-type gastric cancer patients with high expression of the entire signature $(\mathrm{HR}=1.61$; $\operatorname{logRank} P=0.043$ ) (Fig. 8 b). Similar to our IL-33/mast cell activation gene expression signature, a trend for better survival probability was found for high expression of a classical mast cell marker gene signature consisting of KIT, FCER1G, and HDC (encoding Histidine Decarboxylase) $(\mathrm{HR}=$ 1.42; $\operatorname{logRank} P=0.067$ ) (Supplementary Fig. 7a) as well as for ST2-receptor encoding gene IL1RL1 $(\mathrm{HR}=1.57$; logRank $P=$ 0.049) (Supplementary Fig. 7b). In accordance with our mast cell-macrophage hierarchy model, Kaplan-Meier survival analysis for macrophages revealed a survival disadvantage for intestinal-type gastric cancer patients with high expression of an alternatively activated macrophage gene signature (Fig. 8c). On the other hand, high expression of a classically activated gene signature correlates with a higher survival probability (Fig. 8d). Our preclinical data argue strongly for a tumor promoting role of a signaling axis emanating from IL-11-induced, tumor cell- derived IL-33 and the subsequent hierarchical activation cascade of mast cells and tumor-associated macrophages. Collectively, these innate immune cells enable the establishment of the microvasculature required for gastric tumor growth and may provide therapeutically actionable targets (Fig. 8e).

\section{Discussion}

Although conflicting data indicate a dichotomous role of mast cells to either restrict or support tumorigenesis, compelling evidence implicates mast cells in promoting progression and metastasis in many solid malignancies. Retrospective studies on human gastric cancers have suggested an involvement of tumoradjacent submucosal mast cells to late stage disease ${ }^{54}$ and metastasis formation ${ }^{12}$. However, the molecular mechanism by which submucosal mast cells contribute to gastric tumorigenesis, and indeed early stage tumorigenesis, is not understood. Here we demonstrate that submucosal accumulation of mast cells in two 
independent murine models of intestinal-type gastric cancer functionally contributes to tumorigenesis and the maintenance of established tumors and that this coincides with the presence of alternatively activated protumorigenic tumor-associated macrophages. We provide evidence that tumor cell-derived IL-33 stands on the apex of a cascade by which mast cells, through recruitment of tumor-associated macrophages and their support of a vascular network, ensures the growth and maintenance of gastric tumors. Indeed, ablating mast cells or macrophages in tumor-bearing mice either individually with cromolyn or clodrosomes, or together with PLX3397, were associated with a vascular collapse and tumor hypoxia. Importantly, we support the relevance of our functional findings in mice with a correlation in humans where a mast cell activation signature associates with poor patient outcome for intestinal-type gastric cancer.

Within the tumor microenvironment, myeloid cells are among the most important innate immune cells that promote tumor formation and restrict the effect of many forms of therapy, most notable immunotherapy ${ }^{55}$. In particular the plasticity of tumor-associated macrophages has attracted interest as therapeutic opportunities to limit the angiogenic and immune suppressive functions conferred by their alternatively activated endotype ${ }^{3}$. On the other hand, mast cells have been proposed to be an abundant source of VEGF, TGF $\beta$, and other angiogenic factors, as well as matrix metalloproteinase 9 and related stromal remodeling enzymes ${ }^{56}$. Indeed, mast cells are one of the earliest cell types recruited into the tumor microenvironment ${ }^{11,57}$, and progression of gastric cancer correlates with both the accumulation of chymase-positive mast cells and increased microvascular density ${ }^{11,58}$. Functionally, these observations are mirrored by an angiogenic switch proposed to be orchestrated by mast cells during early stages of pancreatic cancer in mice required for the extensive vascular elaboration to prevent hypoxia during expansion of tumors ${ }^{16}$. Surprisingly, the authors in this early study had argued against a role for macrophages in mast cell-dependent induction of early tumor angiogenesis, based on their observations that macrophage recruitment to pancreatic islets remained unaffected by mast cell deficiency. Indeed, our observations that IL33 result in the induction of Vegfa expression in isolated mast cells supports this view and this is corroborated by reduced microvessel density in tumors of $g p 130^{\mathrm{FF}}$; St2 ${ }^{-/-}$mice. However, our observations presented here following either genetic ablation of mast cells or macrophages, or their respective pharmacological inhibition by cromolyn and clodronate or PLX3397 strongly suggest that the endothelial cell support provided by mast cells also depends in part on the recruitment of macrophages.

Because we functionally link tumor growth to the presence of mast cells in two independent genetic models, it is highly unlikely that this arises from independent serendipitous effects of the two models other than mast cells. Thus, it is highly likely that the incompletely understood mechanisms by which cromolyn blocks mast cell degranulation mediated the anti-tumor effect observed here, rather than cromolyn also affecting monocytes, the production of the neutrophil product myeloperoxidase, and circulating growth ${ }^{59}$. Akin to tumor-associated macrophages being induced by tumor cells to adopt an alternatively activated endotype optimized for wound healing, neoplastic epithelia appears to also corrupt the wound healing function of mast cells to facilitate tumor growth ${ }^{55}$. Accordingly, tumor cell-specific overexpression of the master-regulator c-Myc not only results in epithelial hyperproliferation (when coinciding with increased expression of $\mathrm{BH} 3$ survival proteins) but also in expression of chemo-attracting CCL2, CCL3, and CCL5 and concomitant accumulation of mast cells $^{16}$. Here we identify a second mechanism that depends on the
IL-1 cytokine family member IL-33, which acts as a chief regulator of innate immunity and inflammation, and maintains epithelial barrier functions of the intestine. Indeed, IL-33 serves $a$ bona fide alarmin and is released by damaged epithelium as an endogenous danger signal to activate innate immune responses ${ }^{28}$. Accordingly, mast cells and ILC2s respond through the IL-33/ST2 axis to the damaged intestinal mucosa arising from acute injury or infection. Therefore mast cells are found at sites of epithelial recovery ${ }^{60}$, and ILC2 cells release IL-13 to stimulate the intestinal stem cell compartment, respectively ${ }^{61}$. Intriguingly, our experimental lines of evidence from tumors of $g p 130^{\mathrm{FF}} ; S t 2^{-/-}$mice show that their reduced tumor burden correlates with decreased mast cell frequency rather than a change in ILC2 abundance. While not ruling out minor contributions by ILC2, regulatory $\mathrm{T}$ cells or other IL-33 responsive cells, the increased tumor mass in $g p 130^{\mathrm{FF}} ; \mathrm{St}^{-1-}$ mice following adoptive transfer of St2-proficient mast cells, provides definitive functional evidence that mast cells act as the major cell population through which IL-33 promotes tumor growth. Accordingly tumor growth of $g p 130^{\mathrm{FF}}$ mice was susceptible to the highly mast cell-selective cellular deficiency in $g p 130^{F F}$; Cpa3-Cre; Mcl1 ${ }^{f l / f l}$ mice, or to cromolyn-dependent inhibition of mast cell degranulation. Meanwhile, and consistent with the capacity of IL-33 to promote tissue repair and wound healing, IL-33 can also mediate metaplasia of the gastric epithelium ${ }^{62}$.

Although epithelial cells of barrier tissues exposed to the environment are major sources of IL-33, and IL-33 expression is further increased during inflammation and in the tumor epithelium $^{63}$, the exact nature of the factors that induce IL-33 expression and its release remain unclear. For instance TNF, IL-1 $\beta$, TGF $\beta$, prostaglandins, lipopolysaccharides, and other pathogenassociated molecular patterns have all been suggested as drivers for IL-33 expression during intestinal adenomatous polyposis ${ }^{64-66}$. Here, we provide evidence for IL-33 expression in gastric tumor epithelium to also be stimulated by an IL-11/ Stat 3 signaling cascade, which we and others have identified as an absolute requirement for effective growth of gastrointestinal tumors. However, at this stage we can only speculate that subepithelial myofibroblasts and possibly tumor-associated endothelial cells, which have been proposed as sources of IL-11 ${ }^{67}$, may also contribute to epithelial expression of IL-33. Furthermore, because mast cells express gp130, we could not formally exclude the possibility that the presence of the hypermorphic $g p 130^{\mathrm{F}}$ allele in mast cells may promote their proliferation and survival ${ }^{46}$, as this receptor allele augments Stat3 signaling in response to IL-6 family cytokines. However, we noted similar submucosal mast cell accumulation adjacent to tumors in $g p 130^{\mathrm{FF}}$ and in $\operatorname{Tg}(\mathrm{Tff} 1-$ CreERT2); $P i k 3 c a^{\mathrm{H} 1047 \mathrm{R} /+} ;$ Pten $^{\mathrm{fl} / \mathrm{fl}}$ mice, where the latter cohorts express WT gp130. Likewise, reconstitution of $g p 130^{\mathrm{FF}}$ mice with WT bone marrow did not affect mast cell numbers and tumor burden, as would be expected if the $g p 130^{\mathrm{F}}$ allele augmented mast cell activity.

Therapeutic inhibition of IL-33 signaling is gaining interest with anti-ST2 antibodies being trialed for asthma. Given the dichotomous activity of IL-33 in tumor biology, timing of anti-IL33 signaling therapy will be crucial. In the context of gastric cancer, mast cells may not only promote metastasis but also modulate the immunosuppressive tumor environment through the release of IL-1768. Thus, IL-33 signaling provides a target to restrict the tumor promoting activities of the myeloid compartment and may ultimately enable rational combination therapies to alleviate the activity by which myeloid cells limit the antitumor immune response. The linear signaling cascade identified here comprising IL-11/IL-33/mast cells/macrophages/tumor cells should provide complementary molecular and cellular targets for the development of improved cancer therapies. 


\section{Methods}

Study approval. All animal studies were conducted in accordance with all relevant ethical regulations for animal testing and research including the Australian code for the care and use of animals for scientific purposes. All animal studies were approved by the Animal Ethics Committee of the Ludwig Institute for Cancer Research, the Walter and Eliza Hall Institute of Medical Research, or Austin Health.

We have complied with all relevant ethical regulations for work with human participants. Collection and usage of human gastric cancer tissues was approved by the Peter MacCullum Cancer Center Ethics Committee and informed consent was obtained from all subjects.

Mice. Knockin mice $\left(g p 130^{\mathrm{Y} 757 \mathrm{~F} / \mathrm{Y} 757 \mathrm{~F}}\right.$, alias $g p 130^{\mathrm{FF}}$ mice), the compound mutants strains $g p 130^{\mathrm{FF}}$; Stat $3^{+/-39,40}$ and $\mathrm{Tg}\left(\right.$ Tff1-CreERT2); Pik $3 c a^{\mathrm{H} 1047 \mathrm{R} /+}$; $P t e n^{\operatorname{lox} /+}$ as well as wild-type control mice were bred on a mixed C57B6 $\times 129 / \mathrm{Sv}$

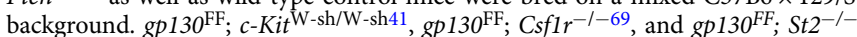
mice ${ }^{70}$, knockout of the Il-33 receptor (encoding gene name: Illrll), gp 130 ${ }^{F F}$; Cpa3Cre; Mcl1 lox/lox44 (mast cell-deficient gp130 13 compound mutant mice) were maintained on a C57B6 background.

$\operatorname{Tg}(T f f 1-C r e E R T 2) ; P i k 3 c a^{\mathrm{H} 1047 \mathrm{R} /+} ;$ Pten ${ }^{\text {lox } /+}$ compound mutant mice were established, where mutant allele induction is induced by the Tff1-CreERT2 transgene and is specific for the gastric epithelium of the stomach ${ }^{71}$.

$g p 130^{\mathrm{FF}}$ mice used in the PLX-treatment experiment were propagated on a C57B6 background.

Cohoused, age- and gender-matched littermates were utilized for all experiments. All strains were housed under specific pathogen-free conditions.

Human gastric cancer tissue. Human gastric cancer tissue micro arrays (TMA) were used to identify toluidine blue-stained mast cells in gastric disease. TMA were established previously ${ }^{72}$. In short, formalin-fixed paraffin-embedded gastric tissue samples we selected, pathology was confirmed by independent pathology review and $0.6 \mathrm{~mm}$ punches were reembedded to generate the TMA. Usage of human gastric cancer tissues was approved by the Peter MacCullum Cancer Center Ethics Committee and informed consent was obtained from all subjects.

Inhibitors and treatment regimes. Cromolyn sodium salt (referred to as cromolyn; Sigma-Aldrich, Cat\# C0399) is a clinically approved mast celldegranulation inhibitor ${ }^{73}$. Cromolyn was dissolved in phosphate buffered saline (PBS) and administered at a concentration of $75 \mathrm{mg} / \mathrm{kg}$ body weight via the intraperitoneal route $(100 \mu \mathrm{l})$ three times per week for 6 consecutive weeks. Control cohorts received PBS.

Clodronate, formulated as liposome-loaded clodrosomes (containing $5 \mathrm{mg}$ clodronate per $1 \mathrm{ml}$ of clodrosome suspension), was injected intraperitoneal as 50 $\mu \mathrm{l}$ clodrosomes suspension twice per week for 6 consecutive weeks. Liposomes containing suspension was injected as placebo in control mice.

The csf1r/c-kit/Flt3-specific inhibitor PLX3397 (Plexxicon), supplied at $800 \mathrm{mg}$ PLX3397 per kg of chow, was given to mice for 4 consecutive weeks represents an estimated dose of $100 \mathrm{mg}$ per kg body weight daily. Vehicle cohorts received unmanipulated chow. For the follow-up cohorts, mice were kept for 4 weeks on unmanipulated chow following their 4 -week-treatment period.

Tissue preparation and processing. Tumors and adjacent antral tissues were resected and weighed and then snap-frozen for RNA or protein isolation. Entire stomachs were removed and fixed in $10 \%$ neutral buffer formalin for histological analysis $^{39}$.

Histological and immunohistochemical analysis. Hematoxylin-Eosin, toluidine blue, Alcian blue, Safranin O, and May-Grünwald-Giemsa staining of formalinfixed paraffin-embedded stomach slides were performed according to theory and practice of histological technique from JD Bancroft ${ }^{74}$.

For in vivo assessment of proliferation by anti-BrdU staining, tissues were collected $2 \mathrm{~h}$ after i.p. injection of $50 \mathrm{mg} / \mathrm{kg}$ BrdU (Amersham Biosciences, GE Healthcare). Stainings for apoptosis (Cell Death Detection Kit, Roche) and tissue hypoxia $(60 \mathrm{mg} / \mathrm{kg} \mathrm{HP1}$ i.p. injection $30 \mathrm{~min}$ prior to tissue collection, detection with Hypoxyprobe-1 Kit, from Hypoxyprobe Inc., USA) were performed according to manufacturers' instructions.

All other immunostaining procedures were conducted as follows. For antigenretrieval, paraffin-embedded sections were either heated in citrate buffer in a microwave pressure cooker ( $\mathrm{pH} 6$ for $15 \mathrm{~min}$ ) or were incubated in $0.1 \%$ trypsin, $3 \%$ acetic acid solution at $37^{\circ}$ for $10 \mathrm{~min}$. Sections were then blocked in $10 \%(\mathrm{v} / \mathrm{v})$ normal goat serum for $1 \mathrm{~h}$ at room temperature. Primary antibodies were diluted in $10 \%(\mathrm{v} / \mathrm{v})$ normal goat serum and incubated overnight at $4{ }^{\circ} \mathrm{C}$ in a humidified chamber. Biotin-labeled secondary antibodies from the Avidin Biotin Complex ABC-kit (Vector Laboratories) were used according to the manufacturer's instructions. Visualization was achieved using 3,3-Diaminobenzine (DAB, DAKO). Images were generated with Aperio ImageScope v11.2.0.780 software. Primary antibodies used: rabbit anti-mouse CD31 (1:200 dilution, Abcam, Cat\# 28364 , RRID: AB_726362), rat anti-mouse F4/80 (1:200 dilution, Abcam, Cat\# 6640, RRID: AB_1140040), rat anti-mouse B220 (1:200 dilution, BD Biosciences, Cat\#
550286, RRID:AB_393581), rabbit anti-mouse CD3 (1:200 dilution, Abcam, Cat\# ab5690, RRID:AB 305055), rat anti-mouse CD8 (1:150 dilution, eBioscience, Cat\# 14-0808-82, RRID: AB_2572861), and rat anti-mouse Foxp3 (1:100 dilution, eBioscience, Cat\# 12-5773-80, RRID: AB_465935).

Immunofluorescence staining. Paraffin-embedded stomach sections were dewaxed, rehydrated and antigen-retrieval was performed by heating in EDTA buffer (pH9) for $15 \mathrm{~min}$ in microwave pressure cooker. Section were incubated for $1 \mathrm{~h}$ at room temperature with goat anti-mouse IL-33 (1:150 dilution, R\&D Systems, Cat\# AF3626, RRID:AB_884269) and then for $1 \mathrm{~h}$ with AlexaFluor 568-conjugated donkey anti-goat secondary antibody (Molecular Probes Cat\# A-11057, RRID: AB 142581). After counter staining with spectral DAPI (1:200 dilution, PerkinElmer, Cat\# FP1490) for 5 min, sections were mounted with Vectashield mounting solution (VECTORlabs, Cat\# H-1400). Imaging was preformed with a Vectra 3.0 Automated Quantitative Pathology Imaging System (PerkinElmer, Cat\# CLS142338) and representative images were produced using Phenochart ${ }^{\mathrm{TM}}$ v1.0.4 software (PerkinElmer).

Quantification. For quantification of mast cell numbers at least three fields of view (10x ocular) of three toluidine blue section per mouse were counted, submucosal area was measured and data represented as mast cells number/area tissue. Similarly, for quantification of CD3, BrdU, and ApopTag staining, either entire tumors or three fields of view of three sections were counted and analyzed comparing to tissue area.

F4/80, CD31, B220, and Hypoxia immune-staining was quantified using Metamorph software (Molecular Devices) or Fiji scripts (ImageJ, htps://Fiji.sc/) determining percentage positive area per area tissue.

Isolation of gastric epithelial cells, immune cells, and flow cytometric analysis. Glandular stomachs or antral tumors were dissected, cut into very small pieces and incubated at $37^{\circ} \mathrm{C}$ for $30 \mathrm{~min}$ in $\mathrm{Ca}^{2+}$ - and $\mathrm{Mg}^{2+}$-free HBSS medium plus $2.5 \%$ FCS and $1 \mathrm{mM}$ EDTA with gentle shaking. Then samples were vortexed for $30 \mathrm{~s}$ and the supernatants containing intraepithelial lymphocytes were separated from the tissue fragments and kept on ice. The remaining tissue samples were further digested in Collagenase/Dispase (Roche) and DNase I (Roche) in $\mathrm{Ca}^{2+}$ - and $\mathrm{Mg}^{2+}$-free HBSS medium plus $2 \% \mathrm{FCS}$ for $45 \mathrm{~min}$ at $37^{\circ} \mathrm{C}$ under continuous rotation. Samples were vortexed for $30 \mathrm{~s}$ once during incubation and once after the incubation. Afterwards, cell suspensions from both incubations were pooled, filtered and washed in PBS plus 5\% FCS for analysis by flow cytometry.

Single cell suspensions were stained for cell surface markers (listed below) and cell viability was controlled with propidium iodide (ThermoFisher Scientific, Cat\# P3566) or SYTOXBlue (ThermoFisher Scientific, Cat\# S34857) staining. Cell sorting was performed with an Aria II cell sorter (BD Bioscience). All cell-type specific flow cytometric gating strategies are presented as a supplementary figure (Supplementary Fig. 8a-e). Mast cells sorting: from $\mathrm{CD}_{4}{ }^{+} \mathrm{EpCam}^{-}$cell population the $\mathrm{CD}_{11} \mathrm{~b}^{-}$cells were selected, then finally the $\mathrm{c}-\mathrm{Kit}^{+} \mathrm{FceRI}^{+}$ population represents the mast cells. Macrophage sorting: from $\mathrm{CD}^{+} 5^{+} \mathrm{EpCam}{ }^{-}$ cells, the $\mathrm{F} 4 / 80^{\mathrm{High}} \mathrm{CD} 11 \mathrm{~b}^{+}$population was selected and back-gated to confirm that the selected macrophage population was $\mathrm{Ly}^{6} \mathrm{C}^{-} \mathrm{Ly}_{6 \mathrm{G}}{ }^{-}$.

The following fluorochrome-conjugated antibodies were used for flow cytometric cell sorting and analysis: CD16/CD32 (1/100 dilution, clone 93, Cat\# 14-0161-86), EpCAM-FITC (1/400 dilution, clone 9CA,Cat\# 11-5791-82), Ly6ceF450 (1/300 dilution, HK1.4, Cat\# 48-5932-82), F4/80-PE-Cy7 (1/400 dilution, BM8, Cat\# 25-4801-82), St2-PerCP-eFluor710 (1:200 dilution, clone RMST2-2, Cat\# 46-9335-82), Foxp3-PE (1/200 dilution, clone FJK-165, Cat\# 12-5773-82), Gata3-PE (1/200 dilution, clone TWAJ, Cat\# 12-9966-42), FceR1-PE-Cy7 (1/300, clone 36951, Cat\# 25-5898-82), and CD3-PE-Cy7 (1/1000, 145-2C11, Cat\# 250031-82) all from Ebioscience; CD11b-PE (1/400 dilution, M1/70, Cat\# 553311), Ly6g (1/300 dilution, 1A8, Cat\# 560602) from BD Pharmingen and CD45.2-A700 (1/400 dilution, clone S450-15-2), CD4 (1/50 dilution, clone GK 1.5), CD11b-PB (1/400 dilution, M1/70), and c-Kit-APC (1/200, ACK-2) from WEHI monoclonal antibody facility.

Isolation and stimulation of BMDM. Bone marrow was collected from the femur and tibia of mice by flushing with sterile PBS. Cells were washed with PBS twice and were filtered through a $100 \mathrm{~mm}$ sieve. Resulting cell suspensions were cultured in DMEM supplemented with $10 \%(\mathrm{v} / \mathrm{v})$ FCS and L929 conditioned medium for 7 days with fresh media changes every second day until fully differentiated into BMDM. After reseeding of cells, alternative macrophage polarization was conducted by stimulation with IL-4 $(20 \mathrm{ng} / \mathrm{ml}$, Preprotech) and IL-13 $(20 \mathrm{ng} / \mathrm{ml}$, Preprotech) for one day.

Cytokine bead array. A total of 50,000 isolated gastric $g p 130^{F F}$ mast cells were cultured in 96-well plates for $3 \mathrm{~h}$ in $30 \mathrm{ng} / \mathrm{mL}$ IL-33. Supernatants were collected, diluted 1:2 in assay buffer and assayed for cytokine concentration using the BioRad Bioplex cytokine bead assay (Bio-Rad Mouse 23-Plex Panel M60009RDPD) strictly according to manufacturer's instructions. 
BMMC adaptive transfer assay. Mast cells were generated from bone marrow from either $g p 130^{F F}$; $S T 2^{+/+}$or $g p 130^{F F}$; $S T 2^{-/-}$mice. Bone marrow was extracted and cells were maintained in IL-3-containing culture medium for several weeks until $>95 \%$ pure BMMC cultures were established. Purity of BMMCs $\left(\mathrm{FceR}^{+}, \mathrm{c}-\right.$ $\mathrm{Kit}^{+}$) was determined via flow cytometry. For the BMMC transplantation assay, syngeneic BMMC from the same colony as the host mice were used. Either $g p 130^{F F} ; S T 2^{+/+}$or $g p 130^{F F}$; ST2 $2^{-/-}$BMMCs were injected into the tail vein of $g p 130^{F F}$; ST2 $2^{-/}$mice. Each mouse received $10^{7}$ BMMC at 5, 8, and 11 weeks of age and final tumor analysis was performed at 14 weeks of age.

Gastric organoid assay. Antral tumors from $g p 130^{F F}$ mice were used to establish tumor-epithelial organoids. Organoids were established and maintained in IntestiCult ${ }^{\mathrm{TM}}$ Organoid Growth Medium (StemCell Technologies) according to manufacturer's protocols. Established organoids were stimulated with $100 \mathrm{ng} / \mathrm{ml}$ IL-11 or PBS control for $4 \mathrm{~h}$ and then processed for gene expression analysis via $\mathrm{qPCR}$. For assessment of organoid growth, PBS or IL-11 stimulated organoids were monitored over 4 days.

RNA isolation and quantitative RT-PCR. Total RNA was extracted from frozen tissue samples using Trizol ${ }^{\circ}$ Reagent (life technologies, Cat\# 15596026) and cDNA was prepared from $2 \mu \mathrm{g}$ RNA using the High capacity cDNA Reverse Transcription kit (Applied Biosystems, Cat\# 4368813) according to the manufacturer's protocol.

From isolated gastric mast cells and from FACS sorted macrophages, RNA was extracted using the RNeasy Plus Micro Kit (QIAGEN, Cat\# 74034) and cDNA synthesis was performed with the ThermoScript ${ }^{\mathrm{TM}} \mathrm{RT}-\mathrm{PCR}$ System (Invitrogen, Cat\# 11146-024) according to the manufacturer's instructions.

Quantitative RT-PCR analyses were performed in technical triplicates with SensiMix SYBR kit (Bioline, Cat\# QT605-20) using the ViiA 7 Real Time PCR System (life technologies). Further details and the sequences of the used oligonucleotides are described in the supplementary methods and Supplementary Table S1.

Kaplan-Meier survival analysis. All Kaplan-Meier survival analysis was performed with KMplot (KMplot.com) ${ }^{53}$. Data from 179 intestinal-type gastric cancer patient were analyzed and following settings were applied: probes = all probe sets, Laurens classification = intestinal-type, Data sets included: GSE14210, GSE15459, GSE22377, GSE29272, and GSE51105, but excluding GSE62254 (as recommended; due to markedly different survival and shifted expression compared to the other data sets). All other settings were kept as default.

Statistics. Unless otherwise stated, data are presented as mean values \pm standard error of the mean $(\mathrm{M} \pm \mathrm{SEM})$. Exact $n$ values are depicted for every data set in the figure legends and they are always true biological replicates, not technical replicates. All data sets were tested for normality with Shapiro-Wilk normality test. Comparisons between two mean values were performed by two-tailed unpaired Student's $t$-test using Prism6 software (GraphPad Software, California, USA). When data sets had significantly different standard deviations according to Prism's $F$-test of variances, Welch correction was applied. $T$-test $t$ values and corresponding degree of freedom (df) are given in figure legends. For comparisons of more than two groups one-way ANOVA test was performed with multiple comparison correction (either Tukey or Bonferroni). ANOVA $F$ values are depicted in each figure legend as F (DFn, Dfd), where DFn is the df nominator and Dfd the df denominator. $P$ values of less than 0.05 were considered statistically significant.

Sample size determination. $G^{*}$ power3.1 software was used to estimate the minimal required sample sizes. Based on previous mean values and SD from previous studies using the $g p 130^{F F}$ mouse model, we calculated a minimal sample size for eight mice per group, when the mean difference is $40 \%$ and the SD $20 \%$ (unpaired two-tailed $t$-test; $\alpha=0.05$, Power $=0.95$ ). Due to difference in availability and frequency of target genotypes born, the number of mice analyzed between cohorts differed. Sample size for subsequent analysis of tissue section staining was limited to number available, as tissues were required for several downstream processes including FACS analysis, RNA and protein expression in addition to histological and immunohistochemical analysis.

Randomization. No formal randomization procedure was performed. However, for genetic mouse experiments, littermates were used to compare different target genotype mice. For in vivo treatment experiments, different treatment groups were equally distributed between mouse litters and mouse cages as well as female and male ratio were kept at 1:1.

Data exclusion. No data was excluded after commencement of quantification. In a few cases, tissue slides were excluded from downstream analysis due to strong background staining or absence of tumor tissue. These slides were excluded from analysis before commencement of quantification and without knowledge of genotypes/treatment details (aka blinded).
Blinding. Assessors were blinded for determining tumor masses and numbers and for quantification of histo- and immunohistochemical staining. Blinding was achieved through labeling minimized to mouse IDs. Genotypes and treatment were only linked back to the mouse ID after completion of quantification.

Reporting summary. Further information on research design is available in the Nature Research Reporting Summary linked to this article.

\section{Data availability}

The Stat3 chromatin immunoprecipitation-sequencing (ChIP-Seq) data have been deposited in the NCBI gene expression omnibus (GEO) under the accession code GSE48285. The source data underlying Figs. 1b, d, 2b-h, 3b-e, 4b-e, 5a-c, 6a, c, d, f, $7 \mathrm{a}-\mathrm{g}$, and $8 \mathrm{a}$ and Supplementary Figs $1 \mathrm{~b}, 2 \mathrm{a}, \mathrm{b}, \mathrm{g}, \mathrm{i}, 3 \mathrm{~d}, 5 \mathrm{a}, \mathrm{b}, \mathrm{g}$, h, and 6e, fare provided as a source data file. All the other data supporting the findings of this study are available within the article and its supplementary information files and from the corresponding author upon reasonable request. A reporting summary for this article is available as a Supplementary Information file.

Received: 23 January 2018 Accepted: 22 May 2019

Published online: 21 June 2019

\section{References}

1. Joyce, J. A. Therapeutic targeting of the tumor microenvironment. Cancer Cell 7, 513-520 (2005)

2. Fridman, W. H., Pagès, F., Sautès-Fridman, C. \& Galon, J. The immune contexture in human tumours: impact on clinical outcome. Nat. Rev. Cancer 12, 298-306 (2012).

3. Qian, B.-Z. \& Pollard, J. W. Macrophage diversity enhances tumor progression and metastasis. Cell 141, 39-51 (2010).

4. Galli, S. J., Nakae, S. \& Tsai, M. Mast cells in the development of adaptive immune responses. Nat. Immunol. 6, 135-142 (2005).

5. Blank, U. \& Rivera, J. The ins and outs of IgE-dependent mast-cell exocytosis Trends Immunol. 25, 266-273 (2004).

6. Galli, S. J., Grimbaldeston, M. \& Tsai, M. Immunomodulatory mast cells: negative, as well as positive, regulators of innate and acquired immunity. Nat. Rev. Immunol. 8, 478-486 (2008).

7. Ribatti, D. et al. Tumor vascularity and tryptase-positive mast cells correlate with a poor prognosis in melanoma. Eur. J. Clin. Investig. 33, 420-425 (2003).

8. Nonomura, N. et al. Decreased number of mast cells infiltrating into needle biopsy specimens leads to a better prognosis of prostate cancer. Br. J. Cancer 97, 952-956 (2007).

9. Strouch, M. J. et al. Crosstalk between mast cells and pancreatic cancer cells contributes to pancreatic tumor progression. Clin. Cancer Res. 16, 2257 (2010).

10. Elpek, G. et al. The prognostic relevance of angiogenesis and mast cells in squamous cell carcinoma of the oesophagus. J. Clin. Pathol. 54, 940-944 (2001).

11. Ribatti, D. et al. Mast cells and angiogenesis in gastric carcinoma. Int. J. Exp. Pathol. 91, 350-356 (2010).

12. Yano, H. et al. Mast cell infiltration around gastric cancer cells correlates with tumor angiogenesis and metastasis. Gastric Cancer 2, 26-32 (1999).

13. Theoharides, T. C. Mast cells and pancreatic cancer. N. Engl. J. Med. 358, 1860-1861 (2008).

14. Nielsen, H. J. et al. Independent prognostic value of eosinophil and mast cell infiltration in colorectal cancer tissue. J. Pathol. 189, 487-495 (1999).

15. Rajput, A. B. et al. Stromal mast cells in invasive breast cancer are a marker of favourable prognosis: a study of 4,444 cases. Breast Cancer Res. Treat. 107, 249-257 (2008).

16. Soucek, L. et al. Mast cells are required for angiogenesis and macroscopic expansion of Myc-induced pancreatic islet tumors. Nat. Med. 13, 1211-1218 (2007).

17. Schonhuber, N. et al. A next-generation dual-recombinase system for timeand host-specific targeting of pancreatic cancer. Nat. Med. 20, 1340-1347 (2014).

18. Lüthi, A. U. et al. Suppression of interleukin-33 bioactivity through proteolysis by apoptotic caspases. Immunity 31, 84-98 (2009).

19. Rickard, J. A. et al. RIPK1 regulates RIPK3-MLKL-driven systemic inflammation and emergency hematopoiesis. Cell 157, 1175-1188 (2014).

20. Martin, N. T. \& Martin, M. U. Interleukin 33 is a guardian of barriers and a local alarmin. Nat. Immunol. 17, 122-131 (2016).

21. Nile, C. J., Barksby, E., Jitprasertwong, P., Preshaw, P. M. \& Taylor, J. J. Expression and regulation of interleukin-33 in human monocytes. Immunology 130, 172-180 (2010). 
22. Kouzaki, H., Iijima, K., Kobayashi, T., O’Grady, S. M. \& Kita, H. The danger signal, extracellular ATP, is a sensor for an airborne allergen and triggers IL33 release and innate Th2-type responses. J. Immunol. 186, 4375-4387 (2011).

23. Ohno, T. et al. Caspase-1, caspase- 8 , and calpain are dispensable for IL-33 release by macrophages. J. Immunol. 183, 7890-7897 (2009).

24. Wang, J.-X. et al. IL-33/ST2 axis promotes mast cell survival via BCLXL. Proc. Natl Acad. Sci. USA 111, 10281-10286 (2014).

25. Licona-Limon, P., Kim, L. K., Palm, N. W. \& Flavell, R. A. TH2, allergy and group 2 innate lymphoid cells. Nat. Immunol. 14, 536-542 (2013).

26. Schiering, C. et al. The alarmin IL-33 promotes regulatory T-cell function in the intestine. Nature 513, 564-568 (2014).

27. Vasanthakumar, A. et al. The transcriptional regulators IRF4, BATF and IL-33 orchestrate development and maintenance of adipose tissue-resident regulatory T cells. Nat. Immunol. 16, 276-285 (2015).

28. Schmitz, J. et al. IL-33, an Interleukin-1-like cytokine that signals via the IL-1 receptor-related protein ST2 and induces T helper type 2-associated cytokines. Immunity 23, 479-490 (2005).

29. Molofsky, A. B., Savage, A. \& Locksley, R. M. Interleukin-33 in tissue homeostasis, injury and inflammation. Immunity 42, 1005-1019 (2015)

30. Gramatzki, D. et al. Interleukin-33 in human gliomas: expression and prognostic significance. Oncol. Lett. 12, 445-452 (2016).

31. Tong, X. et al. Interleukin-33 predicts poor prognosis and promotes ovarian cancer cell growth and metastasis through regulating ERK and JNK signaling pathways.Mol. Oncol. 10, 113-125 (2016).

32. Chen, S.-F. et al. The paracrine effect of cancer-associated fibroblast-induced interleukin-33 regulates the invasiveness of head and neck squamous cell carcinoma. J. Pathol. 231, 180-189 (2013).

33. Maywald, R. L. et al. IL-33 activates tumor stroma to promote intestinal polyposis. Proc. Natl Acad. Sci. USA 112, E2487-E2496 (2015).

34. O'Donnell, C. et al. An antitumorigenic role for the IL-33 receptor, ST2L, in colon cancer. Br. J. Cancer 114, 37-43 (2016).

35. Malik, A. et al. IL-33 regulates the IgA-microbiota axis to restrain IL1a-dependent colitis and tumorigenesis. J. Clin. Investig. 126, 4469-4481 (2016).

36. Eissmann, M. F. et al. Interleukin 33 signaling restrains sporadic colon cancer in an interferon-gamma dependent manner.Cancer Immunol. Res. 6, 409-421 (2018).

37. Thiem, S. et al. mTORC1 inhibition restricts inflammation-associated gastrointestinal tumorigenesis in mice. J. Clin. Investig. 123, 767-781 (2013).

38. Putoczki, TracyL. et al. Interleukin-11 Is the dominant IL-6 family cytokine during gastrointestinal tumorigenesis and can be targeted therapeutically. Cancer Cell 24, 257-271 (2013).

39. Ernst, M. et al. STAT3 and STAT1 mediate IL-11-dependent and inflammation-associated gastric tumorigenesis in gp130 receptor mutant mice. J. Clin. Investig. 118, 1727-1738 (2008)

40. Jenkins, B. J. et al. Hyperactivation of Stat3 in gp130 mutant mice promotes gastric hyperproliferation and desensitizes TGF-beta signaling. Nat. Med. 11, 845-852 (2005).

41. Grimbaldeston, M. A. et al. Mast cell-deficient W-sash c-kit Mutant KitW-sh/ $\mathrm{W}$-sh mice as a model for investigating mast cell biology in vivo. Am. J. Pathol. 167, 835-848 (2005).

42. Wolters, P. J. et al. Tissue-selective mast cell reconstitution and differential lung gene expression in mast cell-deficient KitW-sh/KitW-sh sash mice. Clin. Exp. Allergy 35, 82-88 (2005).

43. Duttlinger, R. et al. W-sash affects positive and negative elements controlling c-kit expression: ectopic c-kit expression at sites of kit-ligand expression affects melanogenesis. Development 118, 705 (1993).

44. Lilla, J. N. et al. Reduced mast cell and basophil numbers and function in Cpa3-Cre; Mcl-1fl/fl mice. Blood 118, 6930-6938 (2011).

45. Traum, D. et al. IL-10-induced gp130 expression in mouse mast cells permits IL-6 trans-signaling. J. Leukoc. Biol. 91, 427-435 (2012).

46. Gyotoku, E. et al. The Il-6 family cytokines, interleukin-6, interleukin-11, oncostatin $\mathrm{M}$, and leukemia inhibitory factor, enhance mast cell growth through fibroblast-dependent pathway in mice. Arch. Dermatol. Res 203, 508-514 (2001).

47. $\mathrm{Li}, \mathrm{X}$. et al. Targeting of tumour-infiltrating macrophages via CCL2/ CCR2 signalling as a therapeutic strategy against hepatocellular carcinoma. Gut 66, 157-167 (2017).

48. Ries, C. H. et al. Targeting tumor-associated macrophages with anti-CSF-1R antibody reveals a strategy for cancer therapy. Cancer Cell 25, 846-859 (2014).

49. Komai-Koma, M. et al. Interleukin-33 amplifies IgE synthesis and triggers mast cell degranulation via interleukin-4 in naïve mice. Allergy 67, 1118-1126 (2012).

50. Belo, A. V. et al. Murine chemokine CXCL2/KC is a surrogate marker for angiogenic activity in the inflammatory granulation tissue. Microcirculation 12, 597-606 (2005).

51. Michalec, L. et al. CCL7 and CXCL10 orchestrate oxidative stress-induced neutrophilic lung inflammation. J. Immunol. 168, 846 (2002).
52. Amann, B., Perabo, F., Wirger, A., Hugenschmidt, H. \& Schultze-Seemann, W. Urinary levels of monocyte chemo-attractant protein-1 correlate with tumour stage and grade in patients with bladder cancer. Br. J. Urol. 82, 118-121 (1998).

53. Szász, A. M. et al. Cross-validation of survival associated biomarkers in gastric cancer using transcriptomic data of 1,065 patients. Oncotarget 7, 49322-49333 (2016).

54. Zhao, Y. et al. Increased numbers of gastric-infiltrating mast cells and regulatory $\mathrm{T}$ cells are associated with tumor stage in gastric adenocarcinoma patients. Oncol. Lett. 4, 755-758 (2012).

55. Engblom, C., Pfirschke, C. \& Pittet, M. J. The role of myeloid cells in cancer therapies. Nat. Rev. Cancer 16, 447-462 (2016).

56. Kanbe, N. et al. Human mast cells produce matrix metalloproteinase 9. Eur. J. Immunol. 29, 2645-2649 (1999)

57. Ranieri, G. et al. Tryptase-positive mast cells correlate with angiogenesis in early breast cancer patients. Int. J. Oncol. 35, 115-120 (2009).

58. Kondo, K. et al. Expression of chymase-positive cells in gastric cancer and its correlation with the angiogenesis. J. Surg. Oncol. 93, 36-42 (2006).

59. Bot, I. et al. Perivascular mast cells promote atherogenesis and induce plaque destabilization in apolipoprotein E-deficient mice. Circulation 115, 2516 (2007)

60. Rigoni, A. et al. Mast cells infiltrating inflamed or transformed gut alternatively sustain mucosal healing or tumor growth. Cancer Res. 75, 3760 (2015).

61. von Moltke, J., Ji, M., Liang, H. E. \& Locksley, R. M. Tuft-cell-derived IL-25 regulates an intestinal ILC2-epithelial response circuit. Nature 529, 221-225 (2016).

62. Petersen, C. P. et al. A signalling cascade of IL-33 to IL-13 regulates metaplasia in the mouse stomach. Gut 67, 805-817 (2017).

63. Liew, F. Y., Girard, J.-P. \& Turnquist, H. R. Interleukin-33 in health and disease. Nat. Rev. Immunol. 16, 676-689 (2016).

64. Kobori, A. et al. Interleukin-33 expression is specifically enhanced in inflamed mucosa of ulcerative colitis. J. Gastroenterol. 45, 999-1007 (2010).

65. Nishida, A. et al. Expression of interleukin 1-like cytokine interleukin 33 and its receptor complex (ST2L and IL1RAcP) in human pancreatic myofibroblasts. Gut 59, 531 (2010).

66. Yanagawa, Y., Suzuki, M., Matsumoto, M. \& Togashi, H. Prostaglandin E2 enhances IL-33 production by dendritic cells. Immunol. Lett. 141, 55-60 (2011).

67. Suen, Y., Chang, M., Lee, S. M., Buzby, J. S. \& Cairo, M. S. Regulation of interleukin-11 protein and mRNA expression in neonatal and adult fibroblasts and endothelial cells. Blood 84, 4125-4134 (1994).

68. Liu, X. et al. Intratumor IL-17-positive mast cells are the major source of the IL-17 that is predictive of survival in gastric cancer patients. PLoS One 9, e106834 (2014)

69. Dai, X.-M. et al. Targeted disruption of the mouse colony-stimulating factor 1 receptor gene results in osteopetrosis, mononuclear phagocyte deficiency, increased primitive progenitor cell frequencies, and reproductive defects. Blood 99, 111 (2002).

70. Hoshino, K. et al. The absence of interleukin 1 receptor-related T1/St2 does not affect T helper cell Type 2 development and its effector function. J. Exp. Med. 190, 1541 (1999).

71. Thiem, S. et al. Stomach-specific activation of oncogenic KRAS and STAT3dependent inflammation cooperatively promote gastric tumorigenesis in a preclinical model. Cancer Res. 76, $2277-2287$ (2016).

72. Boussioutas, A. et al. Distinctive patterns of gene expression in premalignant gastric mucosa and gastric cancer. Cancer Res. 63, 2569-2577 (2003).

73. Thompson, P. J., Hanson, J. M. \& Morley, J. Asthma, mast cells, and sodium cromoglycate. Lancet 322, 848-849 (1983)

74. Bancroft, J. D. \& Alan, S. Theory and practice of histological techniques. 3rd edn, (Churchill Liningstone Elsevier, New York, 1990).

\section{Acknowledgements}

We thank the animal facility staff of La Trobe University and the Ludwig Institute for Cancer Research as well as the Austin and WEHI histology department for technical assistance. We also acknowledge the excellent support for cell sorting by David Baloyan (ONJCRI) and image generation and analysis by Cameron Nowell (Monash University) and Lachlan Whitehead (WEHI). This work was supported through the Victorian State Government Operational Infrastructure Support, the Nation Health and Medical Research Council (NHMRC) of Australia grants 1092788, 1067244, 1069024 and Cancer Council Victoria's Grant-in-Aid 1160708. M.E. is a NHMRC Principal Research Fellow and also received funding from Ludwig Cancer Research.

\section{Author contributions}

Experimental work: M.F.E., A.J., M.B., R.J.J.O’D., T.P., C.D., J.B., S.T., E.T., N.D.H., N.E. A.R.P., and F.M. Data interpretation: M.F.E, A.J., M.B., R.J.J.O’D., A.B., M.L.H., M.A.G., F.M., and M.E. Writing and editing of manuscript: M.F.E., M.L.H., F.M., and M.E. Study concept: M.F.E., A.J., F.M., and M.E. Funding: M.E. 


\section{Additional information}

Supplementary Information accompanies this paper at https://doi.org/10.1038/s41467019-10676-1.

Competing interests: The authors declare no competing interests.

Reprints and permission information is available online at http://npg.nature.com/ reprintsandpermissions/

Peer review information: Nature Communications thanks Jean Marshall and other anonymous reviewer(s) for their contribution to the peer review of this work. Peer reviewer reports are available.

Publisher's note: Springer Nature remains neutral with regard to jurisdictional claims in published maps and institutional affiliations. (c) Open Access This article is licensed under a Creative Commons Attribution 4.0 International License, which permits use, sharing, adaptation, distribution and reproduction in any medium or format, as long as you give appropriate credit to the original author(s) and the source, provide a link to the Creative Commons license, and indicate if changes were made. The images or other third party material in this article are included in the article's Creative Commons license, unles indicated otherwise in a credit line to the material. If material is not included in the article's Creative Commons license and your intended use is not permitted by statutory regulation or exceeds the permitted use, you will need to obtain permission directly from the copyright holder. To view a copy of this license, visit http://creativecommons.org/ licenses/by/4.0/.

(C) The Author(s) 2019 